\title{
Ground Vibration Isolation of Multiple Scattering by Using Rows of Tubular Piles as Barriers
}

\author{
Miao-miao Sun, ${ }^{1}$ Guo-qian Liang, ${ }^{2}$ Tang-dai Xia, ${ }^{3,4}$ Xiu-liang Chen, ${ }^{5,6}$ Jing-lin Qian, ${ }^{5,6}$ \\ Hua-jian Fang, ${ }^{5,6}$ Xue-mei Li, ${ }^{5,6}$ and Xiao-li Wan ${ }^{5,6}$ \\ ${ }^{1}$ Zhejiang University City College, Hangzhou 310015, China \\ ${ }^{2}$ Zhejiang Tongji Vocational College of Science and Technology, Hangzhou 311231, China \\ ${ }^{3}$ Institute of Geotechnical Engineering, Zhejiang University, Hangzhou 310058, China \\ ${ }^{4}$ MOE Key Laboratory of Soft Soils and Geo-Environmental Engineering, Zhejiang University, Hangzhou 310058, China \\ ${ }^{5}$ Zhejiang Institute of Hydraulics and Estuary, Hangzhou 310020, China \\ ${ }^{6}$ Zhejiang Provincial Key Laboratory of Hydraulics Disaster Prevention and Mitigation, Hangzhou 310020, China
}

Correspondence should be addressed to Miao-miao Sun; melvasun@126.com

Received 19 June 2013; Accepted 9 March 2014; Published 7 May 2014

Academic Editor: Vadim V. Silberschmidt

Copyright (C) 2014 Miao-miao Sun et al. This is an open access article distributed under the Creative Commons Attribution License, which permits unrestricted use, distribution, and reproduction in any medium, provided the original work is properly cited.

\begin{abstract}
A new formal solution for the multiple scattering of plane harmonic waves by a group of arbitrary configuration tubular piles in an elastic total space is derived. Each order of scattering satisfies prescribed boundary conditions at the interface of tubular piles, which is delivered as the sum of incident and scattering waves. The first order performs the scattering wave by each scattered pile and the subsequent orders resulted from the excitation of each pile of first order of scattering from the remaining tubular piles. Advanced scattering orders can be regarded as the same manners. Several series of scattering coefficients are figured out with the aids of addition theorem so that the exact steady-state solution for the scattered displacement and stress is obtained. Particularly, when internal diameter of tubular piles tends to be infinitely small, it degenerates to a solid pile problem. By imposing the normalized displacement amplitudes and transmissibility indices, the influences of specific parameters such as scattering orders, internal and external diameter ratio of piles, pile material rigidity, position and distances between tubular pile and pile rows, and pile numbers are discussed. Certain recommended conclusions have been drawn as the guidelines of practical engineering design for discontinuous barrier of tubular piles.
\end{abstract}

\section{Introduction}

The progress of modern society and development brings about a great deal of environmental problems. Traffic, machine operation, and infrastructure construction induced vibration is one of those which is becoming a most talked topic in recent years. Generally, vibration pollutions give rise to not only the uncomfortable residential experiences around neighborhood, but also the overruns of high-tech plants that have strict limit states. In numerous methods of vibration isolation, installing a barrier between vibration sources and the protected equipment or construction appears to be the ideal means. For the past few decades, trenches for screening as research hotspots have already been discussed. Trenches regardless of whether they are in-filled or not serve as a normal perfect way of practical approaches due to their convenience of construction and effective results emerged from the continuous barriers.

However, a series of disadvantages such as high water table levels and field soil instability set limits to the screening in certain circumstances. Thereafter, the alternative method of vibration isolation which is usually considered as the discontinuous barriers by rows of piles is widely adopted in soft soil area. Piles can be driven deeply into soil and arranged in any desirable formation creating wave barriers. Numerous relevant literature researches about using solid piles have been presented during the last several years.

Traditionally from experimental methods, Barkan [1] proposed sheet piles field tests in order to examine the performances creatively. Richart et al. [2] employed piles 
that served as the isolation barrier soon afterwards. Woods et al. [3] investigated the isolation effectiveness of a row of piles as screening by holography technique. They raised a criterion of designing that the piles' diameters should be no less than 0.6 times Rayleigh wavelengths and the net separations between piles should be no more than 0.4 times. Haupt [4] carried out a series of model scale tests on the vibration isolation of various measures including concrete walls in a laboratory ground. Afterwards, Liao and Sangrey [5] inspected the isolating effectiveness by model piles when sound waves propagated in fluid media. They concluded a point of view that flexible piles played better roles than stiff ones. Boroomand and Kaynia [6] presented an approximate analytical foundation model to examine the efficiency of rigid and elastic piles as isolator in half space. Kani and Hayakawa [7] put use to field tests on PC-wall piles to evaluate the reduction of ground vibration.

Unlike the classic empirical approaches, numerical methods were widely utilized recently. Especially the boundary element method seemed to show a good strategy for dynamics response. More detailed descriptions on the barrier systems may be found. Kattis et al. $[8,9]$ put forward a 3D frequency domain BEM analyzing isolated traits of a row of piles. Several patterns of piles which included tubular, solid with circular and square cross-section have been taken as the research objects. The tubular piles were regarded as long cylindrical cavities.

Most of the existing theoretical researches concerning only one row of piles subjected to the incident waves were still not consummate. Avilés and Sánchez-Sesma [10,11] analyzed the properties of one row solid piles acted as the isolator of $\mathrm{SH}, \mathrm{SV}$, and $\mathrm{P}$ waves in an elastic medium. The numerical investigation showed that rigid piles were superior to the flexible ones. The most valid diameter of piles was between 0.25 and 1 wave shear wavelengths. Later on, they developed a theoretical model to study the amplitude reduction behind one row of cylindrical solid piles when Rayleigh waves are incident. They concluded that the optimum efficiency would be gotten when the diameter of piles equaled 0.25 wavelengths and the pile length equaled 2 wavelengths. Gao et al. $[12,13]$ employed the half-space Green's function to inspect 3D multirows of square piles as barrier in a far field. $\mathrm{Xu}$ et al. $[14,15]$ initiated single-row tubular piles as screening barrier utilizing wave function expansion method under the circumstance of P and SV waves' incidence. Cai et al. [16-18] studied the wave scattering by single-row piles in poroelastic soil with the aids of Fourier-Bessel series expansion. Tsai et al. [19] applied a 3D BEM in the frequency domain to investigate the screening effectiveness of circular piles in a row for a massless square foundation subjected to harmonic vertical loading. Xia et al. [20] delivered the analytical solutions of an arbitrary configuration of rigid solid piles as wave scatterers based on the solutions of acoustic and electromagnetic waves scattering by immovable rigid cylinders.

However, in the circumstance that stiffness of piles is much smaller than soil, namely, elastic piles case, the screening effectiveness is often more deteriorated than rigid ones; thus tubular piles are substitutes for solid piles as discontinuous barrier. Not only are they a cost-saving and convenient constructed solution of materials compared to rigid ones, but also they cut off the medium of wave propagation derived from their hollowed formations, even rivaling the continuous barrier.

Some theories of acoustic and electromagnetic waves scattered by shells in elastic medium as the basis of tubular piles issue have been accumulated. More recently, Huang and Gaunaurd [21, 22] denoted a solution of scattering of coherent acoustic waves by two spherical shells. Robert et al. [23] proposed a way of solving the scattering problems by finite cylindrical cavities. Le Bas et al. [24] studied the multiple scattering and fission phenomena by multishell in water.

Nevertheless, to date, the theory of multiple scattering by tubular piles as discontinuous barrier is still immature and deficient. Assumption of scattering orders has remained to be discussed as single from the literatures mentioned above, in which the scatterers are far apart so that their excitation can be taken as the incident waves [25]. However, separations between piles and pile-rows are small enough to guarantee the overall effect actually. In addition, little attention has been paid to analyze tubular piles in elastic medium in which the soil is treated as an isotropic homogeneous solid. Other methodologies have been most adopted by frequency domain boundary element, let alone considered of more general arrangement of piles, most of whose cross-section configurations trends to be identical and the arrangement is linear.

This paper presents a creative method of calculating multiple scattering by an arbitrary configuration and diameter of tubular piles as discontinuous screen-scatters. The response of tubular piles to this excitation is the so-called first-order scattering. In calculating the second-order scattering from one of the scatterers, the first-order radiation of all the others is taken to be the secondary excitation, and the fields of all these remaining tubular piles are translated to the origin of that particular pile from which the second-order scattering is to be calculated. Following this procedure for all the objects present, a recursion relation is obtained for higher order scattered fields in terms of the scattered field of the single, component object, and thus successively higher order corrections are made to the single scattering solution for the configuration. With the aids of addition theorems, boundary conditions both in and out tubular piles have been implemented for each pile and scattering fields of the remaining piles can be expressed with respect to the origin of piles for which the boundary conditions applied. Numerical analyses have been taken to confirm the multiple scattering calculating valid and reasonable for engineering applications.

\section{The Geometry and Theoretical Model}

The geometric position of the scatterers chosen for the theoretical analysis of the problem is shown in Figure 1; the cylindrical tubular piles are located in an isotropic, homogeneous, elastic infinite medium, of which $\lambda$ and $\mu$ are Lamé constants and $\rho$ is matrix mass density. The cylinders extend to infinity with zero displacement along the pile shaft so that the problem can be simplified as a two-dimensional, plane-strain problem. 


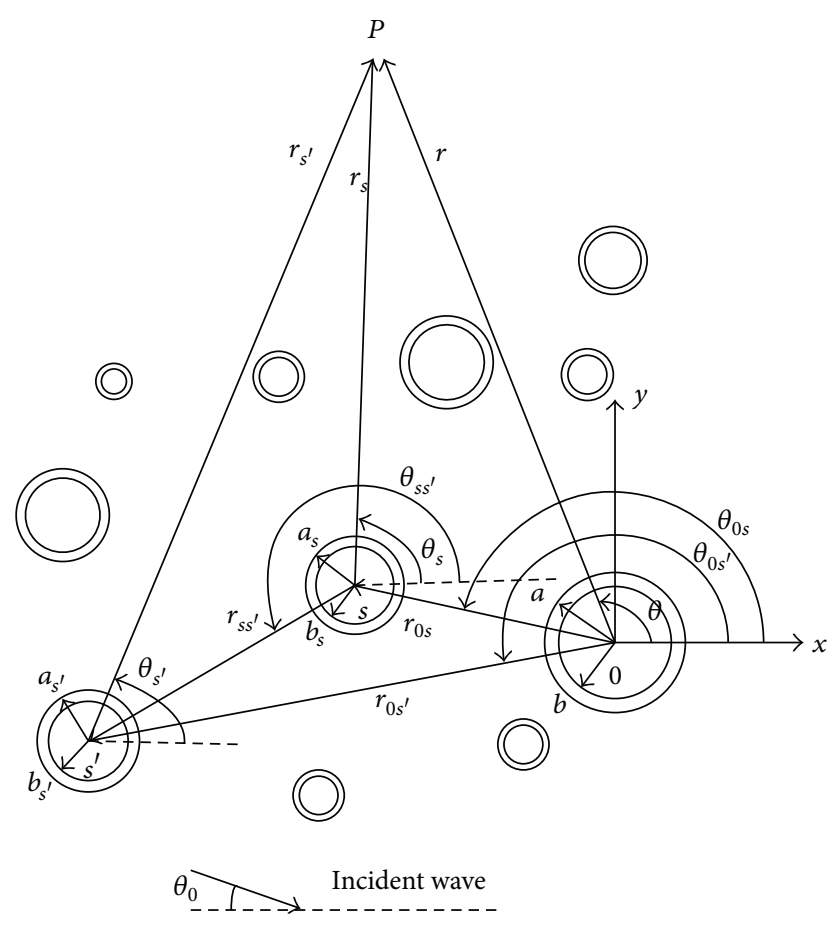

FIGURE 1: Geometric model and analysis coordinate systems.

The reference coordinate system begins with the origin 0 and is set up as a rectangular Cartesian coordinate $(x, y)$ or a polar coordinate $(r, \theta)$. The first tubular pile above the $x$-axis is defined as number 0 ; the next ones are identified by the numbers $s, s^{\prime}, s^{\prime \prime}$, and so forth. The piles are with the external radii $a, a_{s}$, and $a_{s^{\prime}}$ and internal radii $b, b_{s}$, and $b_{s^{\prime}}$, respectively. The point $P$ stands for an arbitrary position with respect to the origin. The distances between point $P$ and pile-centers are $r$, $r_{s}, r_{s s^{\prime}}$ and so forth; the angles independent of each cylinder are $\theta, \theta_{s}, \theta_{s s^{\prime}}$ and so forth; their center-to-center spaces are $r_{0 s}, r_{s s}, r_{s s^{\prime}}$ and so forth.

The multiple scattering procedure can be described as follows. When a series of steady-state harmonic waves propagate from infinite distance with incident angle $\theta_{0}$, the first order of scattering is regarded as the most common "single scattering" hypothesis in which all the scatterings are calculated by translating the primary incident plane waves to the origin of each pile and then summing all the responses of the first excitations. On the basis of the first scattering, second order of scattering is excited by the first scattering waves which perform as the "incident" waves, and the fields of all these remaining piles are translated to the origin of that specific pile. It gives rise to a subsequent recursion relationship for successive orders so that the multiple scattering physical processes made sense.

Equation of motion for the displacement vector $\mathbf{u}$ at the point $P$ is expressed as [26]

$$
(\lambda+2 G) \nabla \nabla \cdot \mathbf{u}-G \nabla \times \nabla \times \mathbf{u}=\rho\left(\frac{\partial^{2} u}{\partial t^{2}}\right),
$$

where $\nabla$ is the gradient operator with $r, \theta$ of polar coordinate or $x, y$ of rectangular Cartesian coordinate system.

The displacement vector $\mathbf{u}$ could be attained by introducing a scalar and a vector potential $\varphi, \psi$, separately,

$$
\mathbf{u}=\nabla \varphi+\nabla \times \psi
$$

where $\varphi$ and $\psi$ are functions of $(x, y)$ or $(r, \theta)$ with time dependence $\exp (-\mathrm{i} \omega t)$.

Substituting $\mathbf{u}$ to the wave equation, (2) turns into

$$
\begin{aligned}
& \nabla^{2} \varphi+\alpha^{2} \varphi=0 \\
& \nabla^{2} \psi+\beta^{2} \psi=0 .
\end{aligned}
$$

Those are known as the Helmholtz equation [27], in which $\alpha^{2}=\omega^{2} / c_{p}^{2}, \beta^{2}=\omega^{2} / c_{s}^{2}, c_{p}=\sqrt{(\lambda+2 G) / \rho}$, and $c_{s}=\sqrt{G / \rho}$ are used to denote wave velocities of $\mathrm{P}$ and $\mathrm{S}$ waves of the matrix. $\omega$ is the so-called circular frequency of harmonic waves, with the corresponding wave numbers $\alpha$ and $\beta$, respectively.

2.1. SH Wave Incidence. In the propagation of $\mathrm{SH}$ wave (the direction vertically polarized), the displacement potential $w$ accords with the Helmholtz equations mentioned above:

$$
\nabla^{2} w+\beta_{s}^{2} w=0
$$

Subscript $s$ of $\beta$ means the wave numbers of soil.

The incident $\mathrm{SH}$ wave displacement potential of unit magnitude is taken as

$$
w^{\text {inc }}=w_{0} \mathrm{e}^{\mathrm{i} \boldsymbol{\beta}_{s} \mathbf{r}},
$$

where inc indicates incident wave, $w_{0}$ is a scalar coefficient, $\mathrm{i}=\sqrt{-1}$, and $t=$ time, with $\mathrm{e}^{-\mathrm{i} \omega t}$ being suppressed. The vector $\boldsymbol{\beta}_{s}$ gives the direction of incidence and $\mathbf{r}$ is the position vector of far field point $P$ with respect to origin 0 .

$\mathrm{SH}$ wave's potentials due to the $m$ th order scattering from sth pile are defined as ${ }^{s} w^{\text {sc }}$ :

$$
s w^{\mathrm{sc}}=\sum_{m=1}^{+\infty} s w_{m}^{\mathrm{sc}}
$$

Thus the total scattering wave field from all piles with number $N$ is constituted as

$$
w^{\mathrm{sc}}=\sum_{s=0}^{N} \sum_{m=1}^{+\infty} s w_{m}^{\mathrm{sc}}
$$

By applying the separation variables method, the scattered field under the polar reference system could be delivered as

$$
w^{\mathrm{sc}}\left(r_{s}, \theta_{s}\right)=\sum_{s=0}^{N} \sum_{m=1}^{+\infty} \sum_{n=-\infty}^{+\infty}{ }^{s} A_{m} \mathrm{H}_{n}^{(1)}\left(\beta_{s} r_{s}\right) \cdot \mathrm{e}^{\mathrm{i} n \theta_{s}},
$$

where the superscript sc denotes scattering waves, $\mathrm{H}_{n}^{(1)}(\cdot)$ is the first kind of Hankel function with order $n$, and ${ }^{s} A_{m}$ is 
the scattering undetermined coefficient of sth pile varying with the multiple order $m$. The total waves become

$$
w=w^{\text {inc }}+w^{\text {sc }}
$$

The tubular elastic piles problem differs from the solid or rigid piles. Therefore the solutions could be readily extended to more general boundary conditions, and expressions could also be obtained for the field inside any pile when it exists.

In consideration of interior transmitted waves which consist of two parts of waves, external and internal propagations, the "transmitted" wave field in the interior of the sth pile is expressed as [28]

$$
w^{t}=\sum_{s=0}^{N} \sum_{m=1}^{+\infty} \sum_{n=-\infty}^{+\infty}\left[{ }^{s} B_{m} \mathrm{H}_{n}^{(1)}\left(\beta_{p} r_{s}\right)+{ }^{s} C_{m} \mathrm{H}_{n}^{(2)}\left(\beta_{p} r_{s}\right)\right] \mathrm{e}^{\mathrm{i} n \theta_{s}},
$$

where ${ }^{s} B_{m}$ and ${ }^{s} C_{m}$ are undetermined transmitted coefficients for the $s$ th pile subject to continuity boundary conditions and $\mathrm{H}_{n}^{(2)}(\cdot)$ is defined as the second kind of Hankel function with order $n$. The subscripts of $\beta$ refer to pile and the rest of the variables refer to the same meaning as mentioned above.

As known to all, incident $\mathrm{SH}$ wave function series expansion equation is

$$
w^{\text {inc }}=w_{0} \mathrm{e}^{\mathrm{i} \boldsymbol{\beta}_{s} \mathbf{r}} \mathrm{e}^{-\mathrm{i} \omega t}
$$

In order to transform generalized wave functions to a specific $s$ pile's coordinate $\boldsymbol{\beta}_{s}, \mathbf{r}_{s}$, through employing the boundary conditions, incident SH wave is expanded to a Fourier-Bessel series in cylindrical wave functions [28]:

$$
w^{\text {inc }}=\mathrm{e}^{\mathrm{i} \beta_{\mathrm{s}} r_{0 s} \cos \left(\theta_{0}+\theta_{0 s}\right)} \sum_{n=-\infty}^{+\infty} \mathrm{J}_{n}\left(\beta_{s} r_{s}\right) \mathrm{e}^{\mathrm{i} n \varphi_{s}},
$$

where $\varphi_{s}=\theta_{s}+\theta_{0}+\pi / 2$, in which the time factor $\mathrm{e}^{-\mathrm{i} \omega t}$ and displacement amplitudes $w_{0}$ in common are both omitted.

The expansion equation of scattering wave function is presented in the same way as listed in (8).

For the sake of solving the undetermined complex coefficients, firstly, It is assumed that the interfaces of soil and piles complied with elastic boundary conditions, which is considered the displacement and stress of interface are continuous and the radial stress of inner boundary is free:

$$
\begin{gathered}
{ }^{s} w_{1}\left(r_{s}, \theta_{s}\right)=\left.{ }^{s} w_{1}^{t}\left(r_{s}, \theta_{s}\right)\right|_{r_{s}=a_{s}}, \\
\left.G_{s} \frac{\partial^{s} w_{1}\left(r_{s}, \theta_{s}\right)}{\partial r_{s}}\right|_{r_{s}=a_{s}}=\left.G_{p} \frac{\partial^{s} w_{1}^{t}\left(r_{s}, \theta_{s}\right)}{\partial r_{s}}\right|_{r_{s}=a_{s}}, \\
\left.G_{p} \frac{\partial^{s} w_{1}^{t}\left(r_{s}, \theta_{s}\right)}{\partial r_{s}}\right|_{r_{s}=b_{s}}=0
\end{gathered}
$$

where $s=0,1,2 \ldots, N, 0 \leq \theta_{s} \leq 2 \pi$, and $G$ defines the shear modulus of soil and pile with the subscripts $s$ and $p$, separately.
Substitute (8), (10), and (12) into (13a), (13b), and (13c); the first order of scattering and transmitted coefficients is obtained:

$$
\begin{aligned}
{ }^{s} A_{1} & =-\frac{\mathrm{J}_{n}^{\prime}\left(\beta_{s} a_{s}\right)-\mathrm{J}_{n}\left(\beta_{s} a_{s}\right){ }^{s} \mathscr{F}_{1}}{\mathrm{H}_{n}^{(1)^{\prime}}\left(\beta_{s} a_{s}\right)-\mathrm{H}_{n}^{(1)}\left(\beta_{s} a_{s}\right){ }^{s} \mathscr{F}_{1}} \mathrm{e}^{\mathrm{i} n\left(\theta_{0}+\pi / 2\right)+\mathrm{i} \beta_{s} \mathbf{r}_{0 s}}, \\
{ }^{s} B_{1}= & \frac{\mathrm{H}_{n}^{(1)^{\prime}}\left(\beta_{s} a_{s}\right) \mathrm{J}_{n}\left(\beta_{s} a_{s}\right)-\mathrm{H}_{n}^{(1)}\left(\beta_{s} a_{s}\right) \mathrm{J}_{n}^{\prime}\left(\beta_{s} a_{s}\right)}{\mathrm{H}_{n}^{(1)^{\prime}}\left(\beta_{s} a_{s}\right)-{ }^{s} F_{1} \mathrm{H}_{n}^{(1)}\left(\beta_{s} a_{s}\right)} \\
& \times \frac{\mathrm{H}_{n}^{(2)^{\prime}}\left(\beta_{p} b_{s}\right)}{\mathrm{H}_{n}^{(1)}\left(\beta_{p} a_{s}\right) \mathrm{H}_{n}^{(2)}\left(\beta_{p} b_{s}\right)-\mathrm{H}_{n}^{(1)^{\prime}}\left(\beta_{p} b_{s}\right) \mathrm{H}_{n}^{(2)}\left(\beta_{p} a_{s}\right)} \\
& \times \mathrm{e}^{\mathrm{i} n\left(\theta_{0}+\pi / 2\right)+\mathrm{i} \beta_{s} \mathbf{r}_{0 s},} \\
{ }^{s} C_{1}= & -\frac{\mathrm{H}_{n}^{(1)^{\prime}}\left(\beta_{s} a_{s}\right) \mathrm{J}_{n}\left(\beta_{s} a_{s}\right)-\mathrm{H}_{n}^{(1)}\left(\beta_{s} a_{s}\right) \mathrm{J}_{n}^{\prime}\left(\beta_{s} a_{s}\right)}{\mathrm{H}_{n}^{(1)^{\prime}}\left(\beta_{s} a_{s}\right)-{ }^{s} F_{1} \mathrm{H}_{n}^{(1)}\left(\beta_{s} a_{s}\right)} \\
& \times \frac{\mathrm{H}_{n}^{(1)^{\prime}}\left(\beta_{p} b_{s}\right)}{\mathrm{H}_{n}^{(1)}\left(\beta_{p} a_{s}\right) \mathrm{H}_{n}^{(2)^{\prime}}\left(\beta_{p} b_{s}\right)-\mathrm{H}_{n}^{(1)^{\prime}}\left(\beta_{p} b_{s}\right) \mathrm{H}_{n}^{(2)}\left(\beta_{p} a_{s}\right)} \\
& \times \mathrm{e}^{\mathrm{i} n\left(\theta_{0}+\pi / 2\right)+\mathrm{i} \beta_{s} \mathbf{r}_{0 s}},
\end{aligned}
$$

where we set

$$
\begin{aligned}
& { }^{s} \mathscr{F}_{1} \\
& =\sqrt{\frac{G_{p} \rho_{p}}{G_{s} \rho_{s}}} \\
& \quad \times \frac{\mathrm{H}_{n}^{(1)^{\prime}}\left(\beta_{p} a_{s}\right) \mathrm{H}_{n}^{(2)^{\prime}}\left(\beta_{p} b_{s}\right)-\mathrm{H}_{n}^{(1)^{\prime}}\left(\beta_{p} b_{s}\right) \mathrm{H}_{n}^{(2)^{\prime}}\left(\beta_{p} a_{s}\right)}{\mathrm{H}_{n}^{(1)}\left(\beta_{p} a_{s}\right) \mathrm{H}_{n}^{(2)^{\prime}}\left(\beta_{p} b_{s}\right)-\mathrm{H}_{n}^{(1)^{\prime}}\left(\beta_{p} b_{s}\right) \mathrm{H}_{n}^{(2)}\left(\beta_{p} a_{s}\right)} .
\end{aligned}
$$

The subscripts $s$ and $p$ of mass density $\rho$ refer to soil and pile.

With regard to the expression of ${ }^{s} \mathscr{F}_{1}$, it is set that when internal radius $b_{s} \rightarrow 0$, so that $\mathrm{H}_{n}^{(1)^{\prime}}\left(\beta_{p} b_{s}\right) \rightarrow 0$, $\mathrm{H}_{n}^{(2)^{\prime}}\left(\beta_{p} b_{s}\right) \rightarrow 0$; meanwhile $G_{r}=G_{p} / G_{s} \rightarrow \infty$; it is inferred that ${ }^{s} \mathscr{F}_{1} \rightarrow \infty$. As a result, ${ }^{s} A_{1}$ degenerates to ${ }^{s} A_{1}=-\mathrm{J}_{n}\left(\beta_{s} a_{s}\right) / \mathrm{H}_{n}^{(1)}\left(\beta_{s} a_{s}\right) \mathrm{e}^{\mathrm{i} n\left(\theta_{0}+\pi / 2\right)+\mathrm{i} \beta_{s} \mathbf{r}_{0 s}}$, which is the same solution form of fixed rigid solid pile [20].

It is demonstrated that the single scattering coefficients are more general. On the other hand, when $b_{s} \rightarrow 0$, thereby Hankel functions $\mathrm{H}_{n}^{(1)^{\prime}}\left(\beta_{p} b_{s}\right) \rightarrow 0$ and $\mathrm{H}_{n}^{(2)^{\prime}}\left(\beta_{p} b_{s}\right) \rightarrow 0$; (15) turns into

$$
s_{\mathscr{F}_{1}}=\sqrt{\frac{G_{p} \rho_{p}}{G_{s} \rho_{s}}} \frac{\mathrm{H}_{n}^{(1)^{\prime}}\left(\beta_{p} a_{s}\right)-\mathrm{H}_{n}^{(2)^{\prime}}\left(\beta_{p} a_{s}\right)}{\mathrm{H}_{n}^{(1)}\left(\beta_{p} a_{s}\right)-\mathrm{H}_{n}^{(2)}\left(\beta_{p} a_{s}\right)} .
$$

Obviously, it degrades a cylindrical solid elastic pile problem [29]; the solutions of tubular pile problem are verified to be correct. 
Since the $(m-1)$ th order of scattering from $s$ th pile is also the excitation for the $m$ th order scattering from the $s^{\prime}$ th, given above with respect to origin $s^{\prime}$, the $s^{\prime}$ th order have to be translated to the origin $s=0$. This translation of the origins for the functions can be accomplished by Graf's addition theorem [30] for the cylindrical wave functions:

$$
\begin{aligned}
\mathrm{H}_{n^{\prime}} & \left(\beta_{s} r_{s^{\prime}}\right) \mathrm{e}^{\mathrm{i} n^{\prime} \theta_{s^{\prime}}} \\
& =\sum_{n=-\infty}^{+\infty} \mathrm{H}_{n-n^{\prime}}\left(\beta_{s} r_{s s^{\prime}}\right) \mathrm{J}_{n}\left(\beta_{s} r_{s}\right) \mathrm{e}^{\mathrm{i} n \theta_{s}-\mathrm{i}\left(n-n^{\prime}\right) \theta_{s s^{\prime}}} .
\end{aligned}
$$

It is taken no account of transmitted waves' superposition as secondary wave source for the result that inner transmitted waves are influenced a little as the excitation of other piles' transmitted waves. Therefore the multiple scattering $(m \geq 2)$ boundary conditions become

$$
\begin{gathered}
{ }^{s} w_{m}\left(r_{s}, \theta_{s}\right) \\
={ }^{s} w_{m}^{s c}+\left.\sum_{s^{\prime}=0, s^{\prime} \neq s}^{N^{\prime}}{ }^{s^{\prime}} w_{m-1}^{s c}\right|_{r_{s}=a_{s}}=\left.{ }^{s} w_{m}^{t}\left(r_{s}, \theta_{s}\right)\right|_{r_{s}=a_{s}}, \\
\left.G_{s} \frac{\partial^{s} w_{m}\left(r_{s}, \theta_{s}\right)}{\partial r_{s}}\right|_{r_{s}=a_{s}}=\left.G_{p} \frac{\partial^{s} w_{m}^{t}\left(r_{s}, \theta_{s}\right)}{\partial r_{s}}\right|_{r_{s}=a_{s}} \\
\left.G_{p} \frac{\partial^{s} w_{m}^{t}\left(r_{s}, \theta_{s}\right)}{\partial r_{s}}\right|_{r_{s}=b_{s}}=0
\end{gathered}
$$

where $s=0,1,2 \ldots, N, 0 \leq \theta_{s} \leq 2 \pi$, and $m \geq 2$.

After a substitution course, the $m$ th order undetermined complex coefficients are given as

$$
\begin{aligned}
& { }^{s} A_{m} \\
& =-\sum_{s^{\prime}=0, s^{\prime} \neq s}^{N^{\prime}} \sum_{n^{\prime}=-\infty}^{+\infty} \frac{\mathrm{J}_{n}^{\prime}\left(\beta_{s} a_{s}\right)-{ }^{s} \mathscr{F}_{m} \mathrm{~J}_{n}\left(\beta_{s} a_{s}\right)}{\mathrm{H}_{n}^{(1)}\left(\beta_{s} a_{s}\right)-{ }^{s} \mathscr{F}_{m} \mathrm{H}_{n}^{(1)}\left(\beta_{s} a_{s}\right)} \\
& \times{ }^{s} \mathscr{H}_{n^{\prime} n}^{(1)}\left(\beta_{s} a_{s s^{\prime}}\right){ }^{s^{\prime}} A_{m-1}, \\
& \sum_{s=0}^{N} \sum_{n=-\infty}^{+\infty}{ }^{s} B_{m} \\
& =\sum_{s^{\prime}=0, s^{\prime} \neq s}^{N^{\prime}} \sum_{n^{\prime}=-\infty}^{+\infty} \sum_{n=-\infty}^{+\infty}\left[\left(\left(J_{n}\left(\beta_{s} a_{s}\right) \mathrm{H}_{n}^{(1)^{\prime}}\left(\beta_{s} a_{\mathrm{s}}\right)\right.\right.\right. \\
& \left.-\mathrm{J}_{n}^{\prime}\left(\beta \mathrm{i}_{s} a_{\mathrm{s}}\right) \mathrm{H}_{n}^{(1)}\left(\beta_{s} a_{s}\right)\right) \\
& \times\left(\mathrm{H}_{n}^{(1)^{\prime}}\left(\beta_{s} a_{s}\right)\right. \\
& \left.\left.-{ }^{s} F_{m} \mathrm{H}_{n}^{(1)}\left(\beta_{s} a_{s}\right)\right)^{-1}\right)
\end{aligned}
$$

$$
\begin{aligned}
\times & \left(\mathrm{H}_{n}^{(2)^{\prime}}\left(\beta_{p} b_{s}\right)\right. \\
\times & \left(\mathrm{H}_{n}^{(1)^{\prime}}\left(\beta_{p} b_{s}\right) \mathrm{H}_{n}^{(2))^{\prime}}\left(\beta_{p} b_{s}\right)\right. \\
& \left.\left.\left.-\mathrm{H}_{n}^{(1)^{\prime}}\left(\beta_{p} b_{s}\right) \mathrm{H}_{n}^{(2)}\left(\beta_{p} a_{s}\right)\right)^{-1}\right)\right] \\
\times{ }^{s} \mathscr{H}_{n^{\prime} n}^{(1)}\left(\beta_{s} a_{s s^{\prime}}\right){ }^{s^{\prime}} A_{m-1}, &
\end{aligned}
$$

$\sum_{s=0}^{N} \sum_{n=-\infty}^{+\infty}{ }^{s} C_{m}$$$
=-\sum_{s^{\prime}=0, s^{\prime} \neq s}^{N^{\prime}} \sum_{n^{\prime}=-\infty}^{+\infty} \sum_{n=-\infty}^{+\infty}\left[\left(\left(J_{n}\left(\beta_{s} a_{s}\right) \mathrm{H}_{n}^{(1)^{\prime}}\left(\beta_{s} a_{s}\right)\right.\right.\right.
$$$$
\left.-\mathrm{J}_{n}^{\prime}\left(\beta_{s} a_{s}\right) \mathrm{H}_{n}^{(1)}\left(\beta_{s} a_{s}\right)\right)
$$$$
\times\left(\mathrm{H}_{n}^{(1)^{\prime}}\left(\beta_{s} a_{s}\right)\right.
$$$$
\left.\left.-{ }^{s} F_{m} \mathrm{H}_{n}^{(1)}\left(\beta_{s} a_{s}\right)\right)^{-1}\right)
$$$$
\times\left(\mathrm{H}_{n}^{(1)^{\prime}}\left(\beta_{p} b_{s}\right)\right.
$$$$
\times\left(\mathrm{H}_{n}^{(1)^{\prime}}\left(\beta_{p} b_{s}\right) \mathrm{H}_{n}^{(2)^{\prime}}\left(\beta_{p} b_{s}\right)\right.
$$$$
\left.\left.\left.-\mathrm{H}_{n}^{(1)^{\prime}}\left(\beta_{p} b_{s}\right) \mathrm{H}_{n}^{(2)}\left(\beta_{p} a_{s}\right)\right)^{-1}\right)\right]
$$$$
\times{ }^{s} \mathscr{H}_{n^{\prime} n}^{(1)}\left(\beta_{s} a_{s s^{\prime}}\right){ }^{s^{\prime}} A_{m-1},
$$

where we set

$$
\begin{aligned}
&{ }^{s} \mathscr{H}_{n^{\prime} n}^{(1)}\left(\beta_{s} r_{s s^{\prime}}\right)=\mathrm{H}_{n-n^{\prime}}^{(1)}\left(\beta_{s} r_{s s^{\prime}}\right) \mathrm{e}^{-\mathrm{i}\left(n-n^{\prime}\right) \theta_{s s^{\prime}}}, \\
& s_{F_{m}}=\sqrt{\frac{G_{p} \rho_{p}}{G_{s} \rho_{s}}[}\left[\left(\mathrm{H}_{n}^{(1)^{\prime}}\left(\beta_{p} a_{s}\right) \mathrm{H}_{n}^{(2)^{\prime}}\left(\beta_{p} b_{s}\right)\right.\right. \\
&\left.-\mathrm{H}_{n}^{(1)^{\prime}}\left(\beta_{p} b_{s}\right) \mathrm{H}_{n}^{(2)^{\prime}}\left(\beta_{p} a_{s}\right)\right) \\
& \times\left(\mathrm{H}_{n}^{(1)}\left(\beta_{p} a_{s}\right) \mathrm{H}_{n}^{(2)^{\prime}}\left(\beta_{p} b_{s}\right)\right. \\
&\left.\left.-\mathrm{H}_{n}^{(1)^{\prime}}\left(\beta_{p} b_{s}\right) \mathrm{H}_{n}^{(2)}\left(\beta_{p} a_{s}\right)\right)^{-1}\right] .
\end{aligned}
$$

It is noted that the operator ${ }^{s} \mathscr{F}_{m}$ is expressed in the same form as ${ }^{s} \mathscr{F}_{1}$. When shear modulus $G_{p} / G_{s} \rightarrow \infty$, the operator of $m$ th order scattering coefficient ${ }^{s} \mathscr{F}_{m} \rightarrow \infty$; meanwhile internal radius $b_{s} \rightarrow 0$ and Hankel functions $\mathrm{H}_{n}^{(1)}{ }^{\prime}\left(\beta_{p} b_{s}\right) \rightarrow 0$ and $\mathrm{H}_{n}^{(2)^{\prime}}\left(\beta_{p} b_{s}\right) \rightarrow 0$. The same degeneration process in which it transforms to fixed rigid 
solid and elastic solid piles' scattering complex coefficients is received. The theoretical solution of total scattering and transmitted waves could be determined finally.

2.2. $P$ and $S V$ Waves Incidence. A coupling scattering phenomenon under $\mathrm{P}$ or SV waves incidence occurs in front of the barrier, and it contains both $\mathrm{P}$ and SV waves. There are of no difference scattering waves functions form between solid pile and tubular pile. However, transmitted waves include both dispersed outward and converged inward stationary waves, which are expressed as expansions ${ }^{s} C_{m} \mathrm{H}_{n}^{(1)}\left(\alpha_{p} r_{s}\right),{ }^{s} D_{m} \mathrm{H}_{n}^{(2)}\left(\alpha_{p} r_{s}\right),{ }^{s} E_{m} \mathrm{H}_{n}^{(1)}\left(\beta_{p} r_{s}\right)$, and ${ }^{s} F_{m} \mathrm{H}_{n}^{(2)}\left(\beta_{p} r_{s}\right)$, where ${ }^{s} C_{m},{ }^{s} D_{m},{ }^{s} E_{m}$, and ${ }^{s} F_{m}$ are transmitted coefficients of $m$ th order and $\alpha, \beta$ are defined as wave numbers of $\mathrm{P}$ and $\mathrm{S}$ waves.

Thus the wave function expansions of incident, scattering, and transmitted waves are

$$
\begin{gathered}
\phi^{\mathrm{inc}}=\mathrm{e}^{\mathrm{i} \alpha_{s} r_{0 s} \cos \left(\theta_{0}+\theta_{0 s}\right)} \sum_{n=-\infty}^{+\infty} \mathrm{J}_{n}\left(\alpha_{s} r_{s}\right) \mathrm{e}^{\mathrm{i} n \varphi_{s}}, \\
{ }^{s} \phi_{m}^{\mathrm{sc}}=\sum_{m=1}^{\infty}{ }^{s} A_{m} \mathrm{H}_{n}^{(1)}\left(\alpha_{s} r_{s}\right) \mathrm{e}^{\mathrm{i} n \varphi_{s}}, \\
{ }^{s} \psi_{m}^{\mathrm{sc}}=\sum_{m=1}^{\infty} \mathrm{i} \cdot{ }^{s} B_{m} \mathrm{H}_{n}^{(1)}\left(\beta_{s} r_{s}\right) \mathrm{e}^{\mathrm{i} n \varphi_{s}}, \\
\phi_{m}^{t}=\sum_{n=-\infty}^{+\infty}\left[{ }^{s} C_{m} \mathrm{H}_{n}^{(1)}\left(\alpha_{p} r_{s}\right)+{ }^{s} D_{m} \mathrm{H}_{n}^{(2)}\left(\alpha_{p} r_{s}\right)\right] \mathrm{e}^{\mathrm{i} n \theta_{s}}, \\
\psi_{m}^{t}=\sum_{n=-\infty}^{+\infty} \mathrm{i}\left[{ }^{s} E_{m} \mathrm{H}_{n}^{(1)}\left(\beta_{p} r_{s}\right)+{ }^{s} F_{m} \mathrm{H}_{n}^{(2)}\left(\beta_{p} r_{s}\right)\right] \mathrm{e}^{\mathrm{i} n \theta_{s}} .
\end{gathered}
$$

The radial and tangential displacements are decomposed:

$$
\begin{aligned}
& u_{r_{s}}\left(r_{s}, \theta_{s}\right)=\frac{\partial \phi}{\partial r_{s}}+\frac{1}{r_{s}} \frac{\partial \psi}{\partial \theta_{s}}, \\
& u_{\theta_{s}}\left(r_{s}, \theta_{s}\right)=\frac{1}{r_{s}} \frac{\partial \phi}{\partial \theta_{s}}-\frac{\partial \psi}{\partial r_{s}} .
\end{aligned}
$$

The stress tensors of $\mathrm{P}$ and $\mathrm{SV}$ waves are expressed as

$$
\sigma=\lambda(\nabla \cdot \mathbf{u}) i+G(\nabla \mathbf{u}+\mathbf{u} \nabla),
$$

where $i$ is unit tensor.

The radial and tangential stress of stress components involved are

$$
\begin{aligned}
\sigma_{r_{s} r_{s}}\left(r_{s}, \theta_{s}\right) & \\
= & \frac{2 G}{r_{s}}\left[-\left(r_{s} \frac{\partial \phi}{\partial r_{s}}+\frac{\partial^{2} \phi}{\partial \theta_{s}^{2}}+\frac{\beta^{2} r_{s}^{2}}{2} \phi\right)+\left(r_{s} \frac{\partial^{2} \psi}{\partial r_{s} \partial \theta_{s}}-\frac{\partial \psi}{\partial \theta_{s}}\right)\right],
\end{aligned}
$$

$$
\begin{aligned}
& \sigma_{r_{s} \theta_{s}}\left(r_{s}, \theta_{s}\right) \\
& =\frac{2 G}{r_{s}}\left[\left(r_{s} \frac{\partial \phi}{\partial r_{s} \partial \theta_{s}}-\frac{\partial \phi}{\partial \theta_{s}}\right)+\left(r_{s} \frac{\partial \psi}{\partial r_{s}}+\frac{\partial^{2} \psi}{\partial r_{s}^{2}}+\frac{\beta^{2} r_{s}^{2}}{2} \psi\right)\right],
\end{aligned}
$$

where the subscripts of all variables refer to the sth pile.

Assuming $m=1$ as the first scattering, the boundary of soil-pile interface at $r_{s}=a_{s}$ satisfies the elastic conditions:

$$
\begin{gathered}
{\left.\left[u_{r_{s}}^{\text {inc }}\left(r_{s}, \theta_{s}\right)+{ }^{s} u_{r_{s}}^{1}\left(r_{s}, \theta_{s}\right)\right]\right|_{r_{s}=a_{s}}=\left.{ }^{t} u_{r_{s}}^{1}\left(r_{s}, \theta_{s}\right)\right|_{r_{s}=a_{s}},} \\
{\left.\left[u_{\theta_{s}}^{\text {inc }}\left(r_{s}, \theta_{s}\right)+{ }^{s} u_{\theta_{s}}^{1}\left(r_{s}, \theta_{s}\right)\right]\right|_{r_{s}=a_{s}}=\left.{ }^{t} u_{\theta_{s}}^{1}\left(r_{s}, \theta_{s}\right)\right|_{r_{s}=a_{s}},} \\
{\left.\left[\sigma_{r_{s} r_{s}}^{\text {inc }}\left(r_{s}, \theta_{s}\right)+{ }^{s} \sigma_{r_{s} r_{s}}^{1}\left(r_{s}, \theta_{s}\right)\right]\right|_{r_{s}=a_{s}}={ }^{t} \sigma_{r_{s} r_{s}}^{1},} \\
{\left.\left[\sigma_{r_{s} \theta_{s}}^{\text {inc }}\left(r_{s}, \theta_{s}\right)+{ }^{s} \sigma_{r_{s} \theta_{s}}^{1}\left(r_{s}, \theta_{s}\right)\right]\right|_{r_{s}=a_{s}}={ }^{t} \sigma_{r_{s} \theta_{s}}^{1},} \\
\left.{ }^{t} \sigma_{r_{s} r_{s}}^{1}\left(r_{s}, \theta_{s}\right)\right|_{r_{s}=b_{s}}=0, \\
\left.{ }^{t} \sigma_{r_{s} r_{s}}^{1}\left(r_{s}, \theta_{s}\right)\right|_{r_{s}=b_{s}}=0,
\end{gathered}
$$

where $s=0,1,2 \ldots, N$ and $0 \leq \theta_{s} \leq 2 \pi$.

Substitute (22)-(24b) and (27a) to (28f); the first scattering coefficients s ${ }^{s} A_{1}$ and ${ }^{s} B_{1}$ and transmitted coefficients ${ }^{s} C_{1}$, ${ }^{s} D_{1},{ }^{s} E_{1}$, and ${ }^{s} F_{1}$ meet the relations below:

$$
\mathbf{P X}=\mathbf{Q},
$$

where $\mathbf{P}$ stands for $6 \times 6$ matrix, $\mathbf{X}$ is the scattering or transmitted coefficients matrix, and $\mathbf{Q}$ presents a vector like this

$$
\begin{gathered}
\mathbf{P}=\left[\begin{array}{cccc}
p_{11} & p_{12} & \cdots & p_{1 m} \\
p_{21} & p_{22} & \cdots & p_{2 m} \\
\vdots & \vdots & \vdots & \vdots \\
p_{n 1} & p_{n 2} & \cdots & p_{n m}
\end{array}\right], \\
\mathbf{X}=\left[\begin{array}{llllll}
{ }^{s} A_{1} & { }^{s} B_{1} & { }^{s} C_{1} & { }^{s} D_{1} & { }^{s} E_{1} & { }^{s} F_{1}
\end{array}\right]^{\mathrm{T}}, \\
\mathbf{Q}=\left[\begin{array}{llll}
q_{1} & q_{1} & \cdots & q_{m}
\end{array}\right]^{\mathrm{T}},
\end{gathered}
$$

where elements $p_{i j}$ and $q_{j}$ indicate $i=1,2, \ldots, n ; j=$ $1,2, \ldots, m$; and $n=m=6$. All the elements in $\mathbf{P}, \mathbf{X}$, and $\mathbf{Q}$ are listed in the Appendix.

Matrices can be solved by Cramer rule only with substituting vector $\mathbf{Q}$ into every column of $\mathbf{P}$ and renewing them so that the first scattering and transmitted coefficients are ${ }^{s} A_{1}=\left|\mathbf{P}_{1} / \mathbf{P}\right|,{ }^{s} B_{1}=\left|\mathbf{P}_{2} / \mathbf{P}\right|,{ }^{s} C_{1}=\left|\mathbf{P}_{3} / \mathbf{P}\right|,{ }^{s} D_{1}=\left|\mathbf{P}_{4} / \mathbf{P}\right|$, ${ }^{s} E_{1}=\left|\mathbf{P}_{5} / \mathbf{P}\right|$, and ${ }^{s} F_{1}=\left|\mathbf{P}_{6} / \mathbf{P}\right|$.

The same degeneration process will be prone to be received when $G_{r}=G_{p} / G_{s} \rightarrow \infty$ and $b_{s} \rightarrow 0$; transmitted coefficients ${ }^{s} C_{1},{ }^{s} D_{1},{ }^{s} E_{1}$, and ${ }^{s} F_{1}$ are equal to 0 , which represents that there is no transmitted field when screen consists of rigid solid piles [20]; on the other side, when $b_{s} \rightarrow a_{s}$, it is equivalent to a cavity problem. 


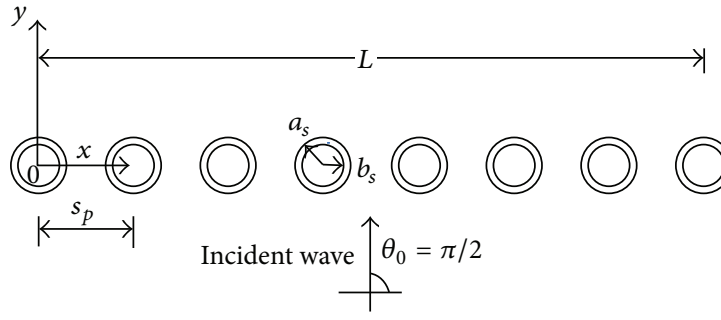

(a) Single-row

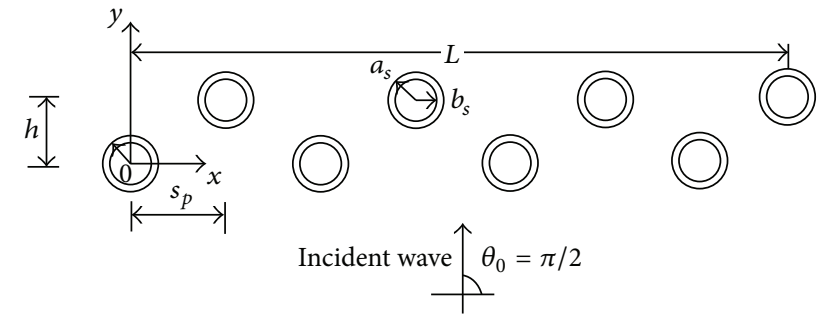

(b) Double-row (hexagon)

FIGURE 2: The reference system and analysis model of incident plane waves scattering by rows of piles $(N=8)$.

Similarly, the elastic boundary conditions of $m$ th $(m \geq 2)$ order are

$$
\begin{aligned}
& {\left.\left[s u_{r_{s}}^{m}\left(r_{s}, \theta_{s}\right)+\sum_{s^{\prime}=0, s^{\prime} \neq s}^{N^{\prime}}{ }^{\prime} s^{\prime} u_{r_{s^{\prime}}}^{m-1}\left(r_{s^{\prime}}, \theta_{s^{\prime}}\right)\right]\right|_{r_{s}=a_{s}}} \\
& =\left.{ }^{t} u_{r_{s}}^{m}\left(r_{s}, \theta_{s}\right)\right|_{r_{s}=a_{s}}, \\
& {\left.\left[s u_{\theta_{s}}^{m}\left(r_{s}, \theta_{s}\right)+\sum_{s^{\prime}=0, s^{\prime} \neq s}^{N^{\prime}}{ }^{\prime} s^{\prime} u_{\theta_{s}}^{m-1}\left(r_{s^{\prime}}, \theta_{s^{\prime}}\right)\right]\right|_{r_{s}=a_{s}}} \\
& =\left.{ }^{t} u_{\theta_{s}}^{m}\left(r_{s}, \theta_{s}\right)\right|_{r_{s}=a_{s}}, \\
& {\left.\left[s \sigma_{r_{s} r_{s}}^{m}\left(r_{s}, \theta_{s}\right)+\sum_{s^{\prime}=0, s^{\prime} \neq s}^{N^{\prime}}{ }^{\prime} s^{\prime} \sigma_{r_{s} r_{s}}^{m-1}\left(r_{s^{\prime}}, \theta_{s^{\prime}}\right)\right]\right|_{r_{s}=a_{s}}} \\
& =\left.{ }^{t} \sigma_{r_{s} r_{s}}^{m}\left(r_{s}, \theta_{s}\right)\right|_{r_{s}=a_{s}}, \\
& {\left.\left[{ }^{s} \sigma_{r_{s} \theta_{s}}^{m}\left(r_{s}, \theta_{s}\right)+\sum_{s^{\prime}=0, s^{\prime} \neq s}^{N^{\prime}}{ }^{\prime} s^{\prime} \sigma_{r_{s} r_{s}}^{m-1}\left(r_{s^{\prime}}, \theta_{s^{\prime}}\right)\right]\right|_{r_{s}=a_{s}}} \\
& =\left.{ }^{t} \sigma_{r_{s} \theta_{s}}^{m}\left(r_{s}, \theta_{s}\right)\right|_{r_{s}=a_{s}}, \\
& \left.{ }^{t} \sigma_{r_{s} r_{s}}^{m}\left(r_{s}, \theta_{s}\right)\right|_{r_{s}=b_{s}}=0 \text {, } \\
& \left.{ }^{t} \sigma_{r_{s} \theta_{s}}^{m}\left(r_{s}, \theta_{s}\right)\right|_{r_{s}=b_{s}}=0 \text {, }
\end{aligned}
$$

where $s=0,1,2 \ldots, N, 0 \leq \theta_{s} \leq 2 \pi$, and $m \geq 2$.

Thus the formula of $m$ th order $(m \geq 2)$ and $(m-1)$ th coefficients is displayed:

$$
\mathbf{P X}=\mathbf{R Y},
$$

where the implications of $\mathbf{P}$ and $\mathbf{Q}$ are no different from former ones, $\mathrm{Y}$ defines a realized $(m-1)$ th coefficients matrix, and $\mathbf{R}$ is $n \times m$ vector. Expressions yield

$$
\begin{aligned}
& \mathbf{X}=\left[\begin{array}{llllll}
{ }^{s} A_{m} & { }^{s} B_{m} & { }^{s} C_{m} & { }^{s} D_{m} & { }^{s} E_{m} & { }^{s} F_{m}
\end{array}\right]^{\mathrm{T}}, \\
& \mathbf{R}=\left[\begin{array}{cccc}
r_{11} & r_{12} & \cdots & r_{1 m} \\
r_{21} & r_{22} & \cdots & r_{2 m} \\
\vdots & \vdots & \vdots & \vdots \\
r_{n 1} & r_{n 2} & \cdots & r_{n m}
\end{array}\right], \\
& \mathbf{Y}=\left[\begin{array}{ll}
{ }^{s} A_{m-1} & { }^{s} B_{m-1}
\end{array}\right]^{\mathrm{T}},
\end{aligned}
$$

where the subscripts of elements $r_{i j}$ are $i=1,2, \ldots, n ; j=$ $1,2, \ldots, m$ and $n=6, m=2$. The rest of the elements are attached in the Appendix. The degeneration process is omitted for abbreviation.

For incidence of SV waves, the only distinction is to substitute incident $\mathrm{P}$ wave for SV wave functions (12) at the right-hand side.

The first coefficients expressions of incident SV waves meet

$$
\begin{gathered}
\mathbf{P X}=\mathbf{S}, \\
\mathbf{X}=\left[\begin{array}{llllll}
{ }^{s} A_{1} & { }^{s} B_{1} & { }^{s} C_{1} & { }^{s} D_{1} & { }^{s} E_{1} & { }^{s} F_{1}
\end{array}\right]^{\mathrm{T}}, \\
\mathbf{S}=\left[\begin{array}{lllll}
s_{1} & s_{2} & \cdots & s_{m}
\end{array}\right]^{\mathrm{T}},
\end{gathered}
$$

where detailed expressions also can be got afterwards and the $m$ th order solutions are no longer different from $\mathrm{P}$ waves.

\section{Numerical Analysis on Screening Effectiveness by Rows of Tubular Piles}

In this study, the surrounding soil is considered as a homogeneous, isotropic medium, the piles' lengths are assumed infinite, and herein this issue goes to a total-space problem. Assuming a series of time-harmonic plane waves with incident angle $\theta_{0}=\pi / 2$ which propagates perpendicularly to this pile group, a numerical calculation model of one row parallel tubular piles with circular cross-section is depicted in Figure 2(a), a rectangular coordinate system is placed from the left, the length of barrier system is $L$, and every tubular 
pile has identical inner diameter $b_{s}$ and external diameter $a_{s}$, while the separations from center to center of piles are equally spaced with $s_{p}$. Some other notations are given as follows:

shear modulus ratio $G_{r}=G_{p} / G_{s}\left(G_{p}\right.$ and $G_{s}$ are shear modulus of pile and soil, separately),

mass density ratio $\rho_{r}=\rho_{s} / \rho_{p}$.

Frequencies of incident $\mathrm{P}$ and $\mathrm{S}$ wave are normalized as

$$
\begin{gathered}
\eta_{p s}=\frac{2 a_{s}}{\lambda_{p s}}=\frac{\alpha_{s} a_{s}}{\pi}, \\
\eta_{s s}=\frac{2 a_{s}}{\lambda_{s s}}=\frac{\beta_{s} a_{s}}{\pi}, \\
\eta_{p p}=\frac{2 a_{s}}{\lambda_{p p}}=\frac{\alpha_{p} a_{s}}{\pi}, \\
\eta_{s p}=\frac{2 a_{s}}{\lambda_{s p}}=\frac{\beta_{p} a_{s}}{\pi},
\end{gathered}
$$

where the first letter in subscripts of frequency $\eta$ or wave length $\lambda$ represents incident $\mathrm{P}$ or $\mathrm{S}$ waves. The second letter in subscripts and the ones in wavenumbers refer to soil or pile. Wavenumbers in different medium meet the relations below:

$$
\begin{aligned}
& \left(\frac{\alpha_{s}}{\beta_{s}}\right)^{2}=\frac{\left(1-2 v_{s}\right)}{2\left(1-v_{s}\right)}, \\
& \left(\frac{\alpha_{p}}{\beta_{p}}\right)^{2}=\frac{\left(1-2 v_{p}\right)}{2\left(1-v_{p}\right)},
\end{aligned}
$$

where $v_{s}$ and $v_{p}$ are Poisson ratios of soil and tubular pile.

Other two-row piles with hexagon appearance are also shown in Figure 2(b), with the pile-row distance $h$. To testify the correctness of multirow applications, one can take the single-row as a special situation of Figure 2(b) while the space between rows of piles $h$ degenerates to 0 . Computations over a wide range of complex arguments require sufficient accuracy only if the Bessel function expansion order $n$ truncated at no less than 8 [29]. Besides, normalized displacement amplitudes $\left|u / u_{0}\right|,\left|v / v_{0}\right|$, and $\left|w / w_{0}\right|$ are employed in numerical calculation, so as all other geometrical parameters.

3.1. Influences of Scattering Orders. Figure 3 illustrates several curves of displacement amplitudes versus distance under different scattering orders. $m$ varies from 1 to 4 . From these figures, it is observed that the same amplitude amplified phenomenon emerges at the front of isolation barrier. The numerical results indicate that a much smaller increment comes up when orders of scattering increase. However, the disparity between single and multiple orders cannot be neglected for the reason that there is coherent phase interference in the successive orders. $m=4$ will be used in the numerical analysis owing to these two curves of $m=3$ and $m=4$ are almost superposed with the incidence of both $S$ and P. It might be attributed to the presence of the dissipation effect. This result, which is consistent with the result of Sun [29] using the same multiple scattering hypothesis in rigid solid pile, is a convincing fact of this research method. It is interpreted that when the elastic wave propagates through the pile barrier, there exists an interference effect due to the wave scattering. The scattering waves are stronger in the center of the pile barrier than at the edge, where more wave energy is transmitted to the screened zone because of a finite size of the barrier. These infinite series' convergence resulted from the scattered waves' diminishing along with the higher order of scattering. A comparison between Figures 3(a) and 3(b) indicates that gapping away each tubular pile from front to back properly causes a prominent improvement of displacement amplitudes reduction along the midline of barrier. In other words, the double-row system decreases no less than 2 times incident waves' amplitude at the range of $100 \leq y / a_{s} \leq 300$ under the same number of piles as singlerow. A further detailed parametric study has been taken as follows.

3.2. Influences of Different Position behind the Barrier. In order to investigate the displacement representation of different spot at the back of pile row, the variations of $\left|w / w_{0}\right|$ and $\left|u / u_{0}\right|$ with varied positions versus $y$-axis by single or double-rows of tubular piles are presented in Figure 4. It is demonstrated that the closer distance it is away from the midline of barrier, the superior responses of amplitudes reduction it will get. In the examination of single elastic row tubular piles in Figures 4(a) and 4(c) under the incident $\mathrm{SH}$ and $\mathrm{P}$ waves, similar characters versus yare received. In the center, it reacts $1 / 3$ displacement amplitudes of the barrier edge within the bounds of $50 \leq y / a_{s} \leq 100$. However, delicate distinction can be differentiated in various positions for $y / a_{s} \geq 200$ due to oscillation verging to steady for distance. Either the rise of shear modulus ratio or pile row distances (a process of tubular piles degrading to rigid solid piles) achieves the remarkable decrease of amplitude in Figures 4(b) and 4(d). The best isolated zone moves back to $100 \leq y / a_{s} \leq 200$. These appearances agree with subsequent parametric studies.

3.3. Influences of Tubular Pile Wall Thickness Ratio $b_{s} / a_{s}$. The tubular pile wall thickness ratio is a unique characteristic to investigate screening effect of the barrier, which has not been taken into account in previous studies. Figure 5 depicts the curves of the midline amplitudes reduction along with the variation of tubular piles' wall thickness ratio $b_{s} / a_{s}$. It can be observed that the maximum reduction occurs in the range $50 \leq y / a_{s} \leq 200$ behind the isolation system. The displacement amplitudes decrease to 0.3 in Figure 5(a) due to the wall thickness attenuating to $1 / 10\left(b_{s} / a_{s}=0.9\right)$ of external diameter, which amounts to $3 / 5$ displacement amplitudes of solid piles barrier $\left(b_{s} / a_{s}=0\right)$. The parallel advantages can be perceived in Figures 5(c) and 5(d) at the range backing to $y / a_{s} \geq 170$ when it is of incident $\mathrm{P}$ waves. For double-row piles, evident decrease of displacement is obtained while the thickness wall reduces. Besides, comparing Figure 5(a) with 


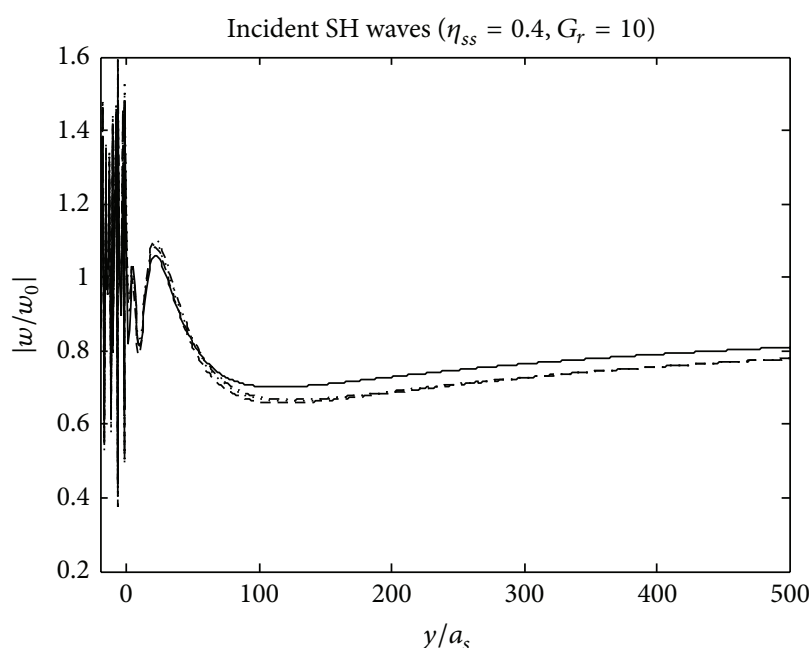

(a) $h=0$

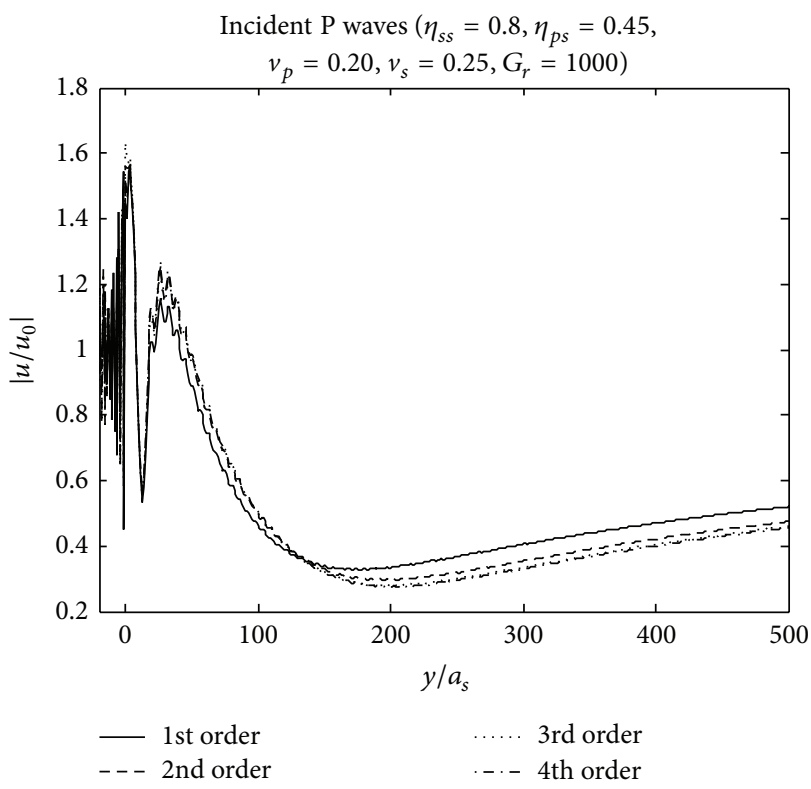

(c) $h=0$

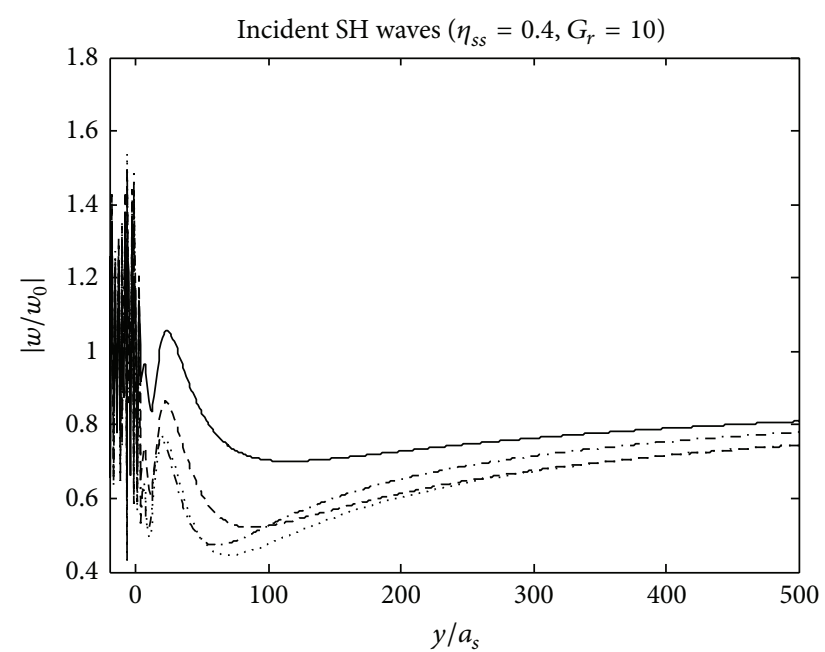

(b) $h=3.0 a_{s}$

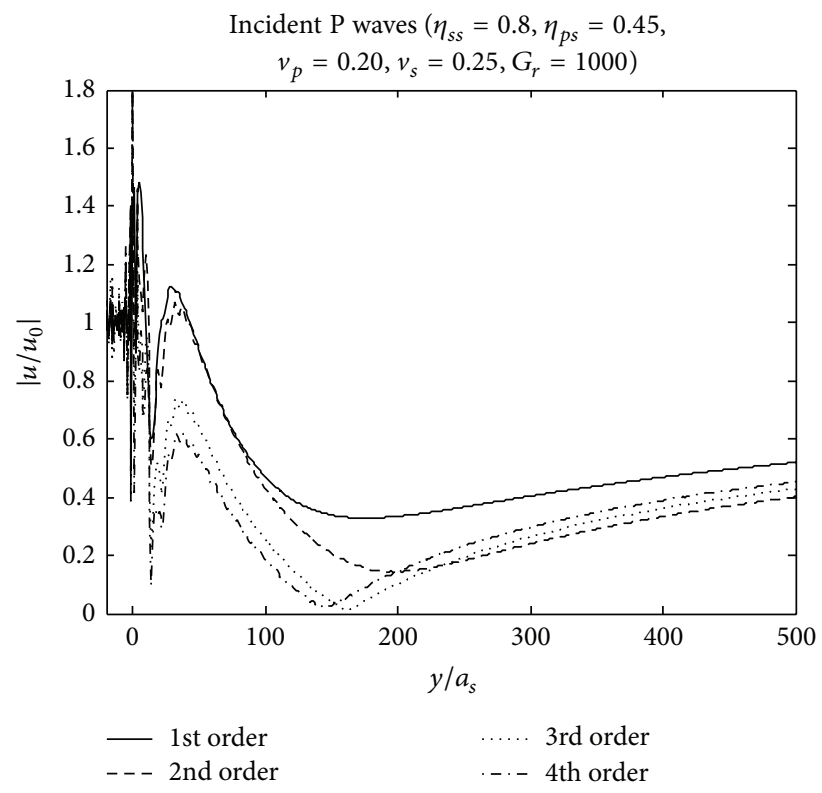

(d) $h=3.0 a_{s}$

FIGURE 3: Normalized displacement amplitudes curves along midline behind the isolation system under different scattering orders by rows of tubular piles $\left(s_{p},=3.0 a_{s}, b_{s}=0.9 a_{s}, 1 \leq m \leq 4, N=8\right)$.

Figure 5(b) or Figure 5(c) with Figure 5(d), it appears that double-row reflects better than single-row. With the same material utilization amount, tubular piles present to have more efficiently isolated achievements than traditional solid piles do. In this case, tubular piles save much cost and have high application value in practical engineering. Thus ratio $b_{s} / a_{s}=0.9$ is taken for analyses below

3.4. Influences of Shear Modulus Ratio $G_{r}$. Different from the rigid solid piles, the tubular piles have another significant factor called shear modulus ratio which evaluates the flexibility of pile body materials and soil properties. Figure 6 presents a series of contours following the $G_{r}$ changed from
10 to infinite when it is under both $\mathrm{SH}$ and $\mathrm{P}$ waves incidence. It is noted that all optimum screening regions are at the center of the isolated scope. Those figures reveal that the minimum values of the normalized displacement amplitudes decrease with the increasing of shear modulus ratio $G_{r}$. As $G_{r}$ varied to 100 when it is of SH waves incidence, the best performance area draws back to $70 \leq y / a_{s} \leq 200$ other than $50 \leq$ $y / a_{s} \leq 100$ when $G_{r}$ equals 10 . The isolation effectiveness performs to reduce $80 \%$ displacement amplitudes meanwhile. When $G_{r}$ grows from 1000 to $+\infty$, it can be observed that further increasing of shear modulus ratio appears to have little impact on the screening effectiveness. The shapes of the contour containing are approximately the same. That is to say, it almost equates with the solid piles under the 


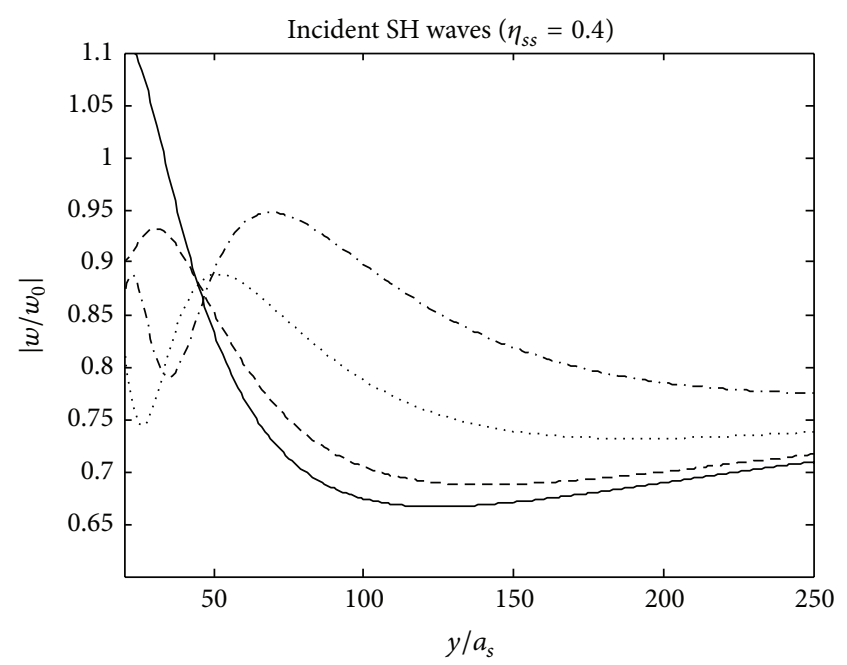

(a) $h=0, G_{r}=10$

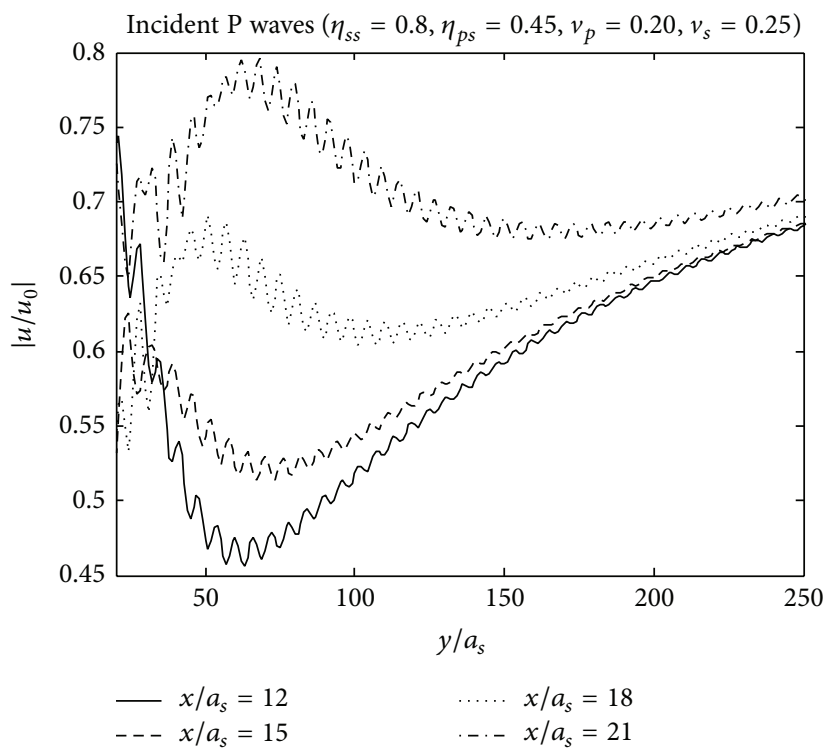

(c) $h=0, G_{r}=10$

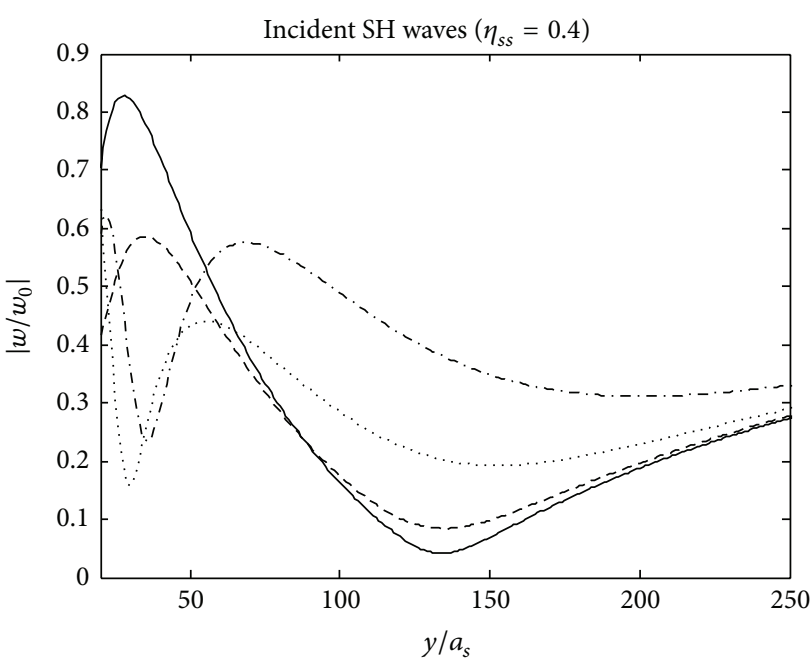

(b) $h=3.0 a_{s}, G_{r}=1000$

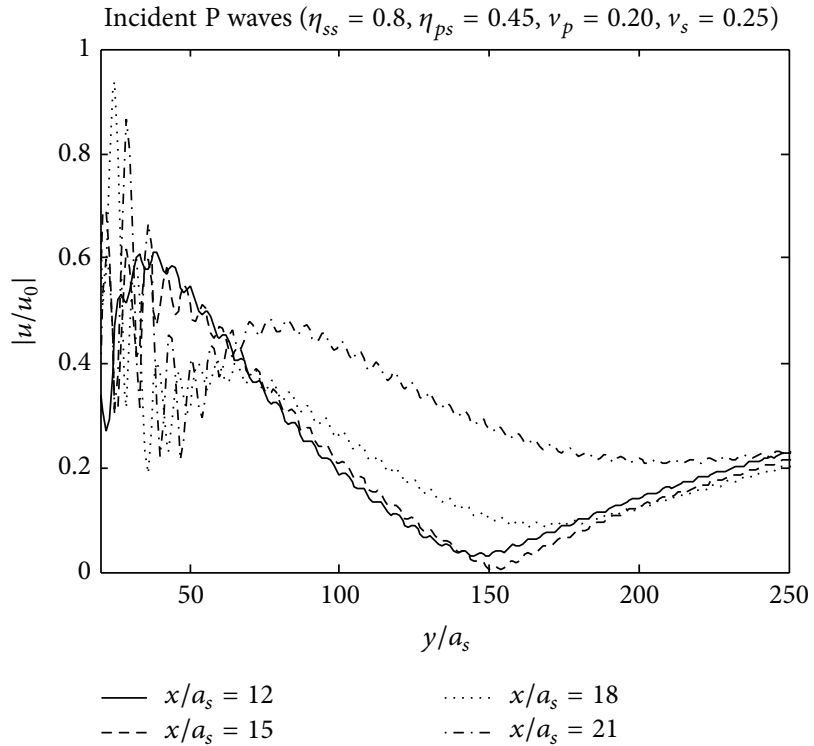

(d) $h=3.0 a_{s}, G_{r}=1000$

FIGURE 4: Normalized displacement amplitudes at different positions behind the barrier $\left(s_{p}=3.0 a_{s}, b_{s}=0.9 a_{s}, m=4, N=8\right)$.

circumstance that $G_{r}$ is greater than 1000. The degeneration characteristics are proved that the numerical algorithm is valid. In other words, elastic tubular piles are far better formation of discontinuous barrier than rigid solid piles. The same tendency can be received in Figures 6(e)-6(h) when it is of $\mathrm{P}$ waves incidence.

3.5. Influences of Separations $s_{p}$ between Each Tubular Pile. Figure 7 depicts the variation of spacing between the tubular piles behind the isolation system. Generally, for all the curves at the distances from the pile unity, they reveal the consistent variation trends in which the closer it gets near the core behind the barrier, the superior reduction of displacement amplitudes it manifests. The normalized value of separation varies from 3.0 to 5.5 , respectively. One can observe that considerable decrease appears with the separation rises. With 3 times of pile diameter, Figure 7 suggests that the value of $\left|w / w_{0}\right|$ or $\left|u / u_{0}\right|$ acquires no less than $50 \%$ incident wave is isolated compared to the pile-row with the normalized separation of 5.0. The rational explanation is that the vibration isolation barrier behaves as an integrated obstacle when the separation is within a certain limit. Along with the separations from pile to pile setting farther away, the pile group displays nearly a series of independent piles instead of a whole unit. However, the effectiveness verges to convergence at the fringe of barrier. As expected, it is noticed that a far field point at $x$-axis except $y / a_{s}$ varying puts up better performance at nearer limits $\left(y / a_{s}=150\right)$ than a remote 


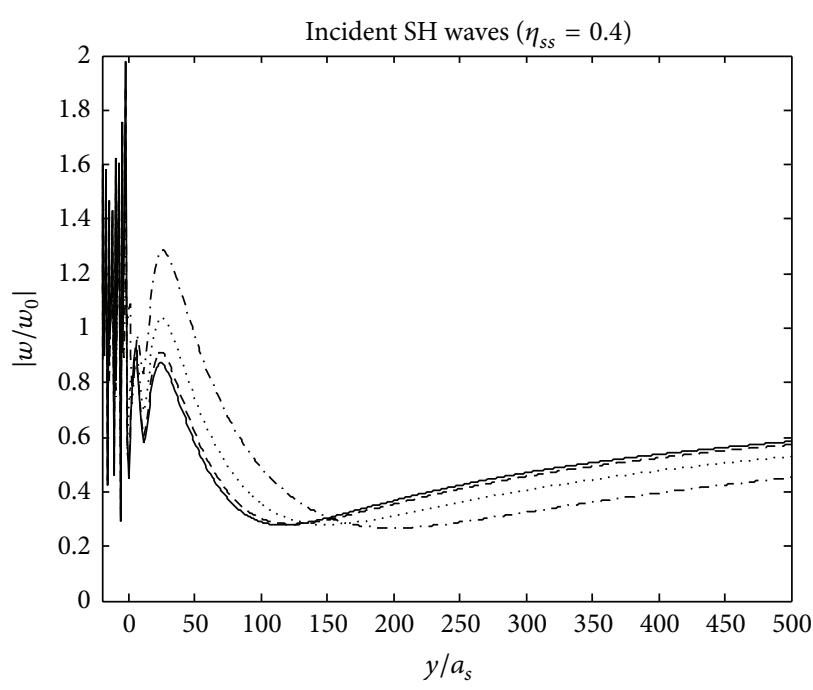

(a) $h=0$

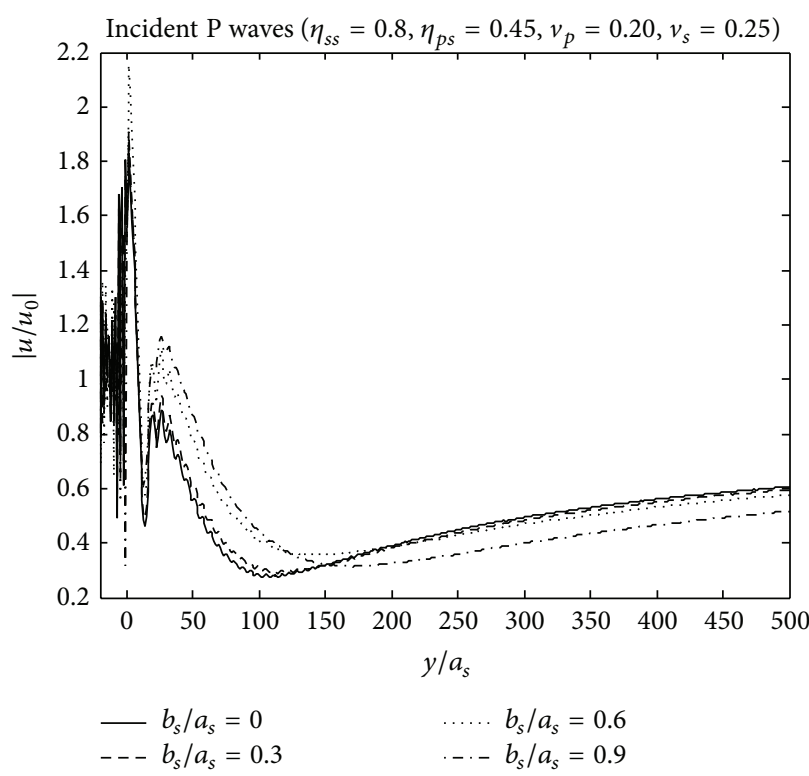

(c) $h=0$

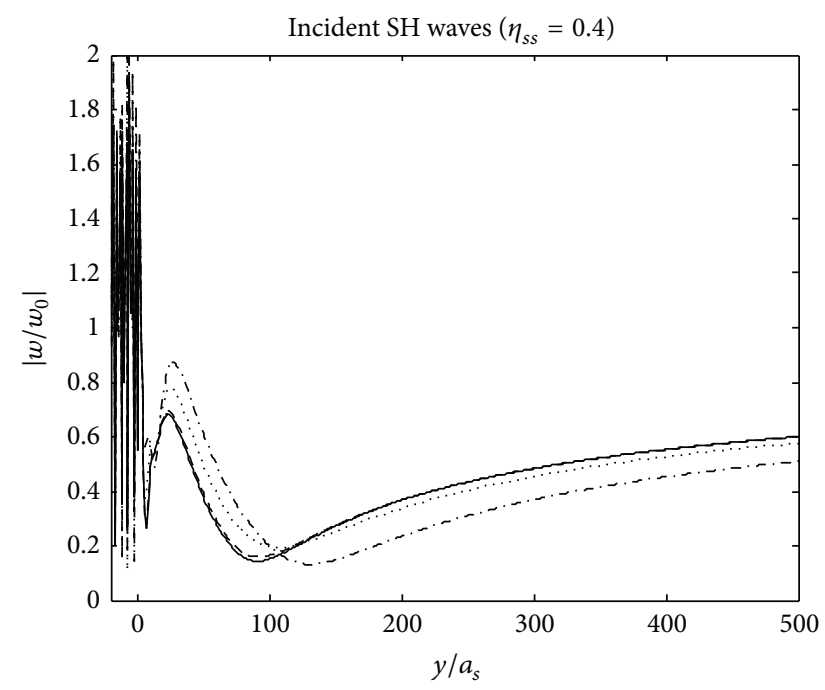

(b) $h=3.0 a_{s}$

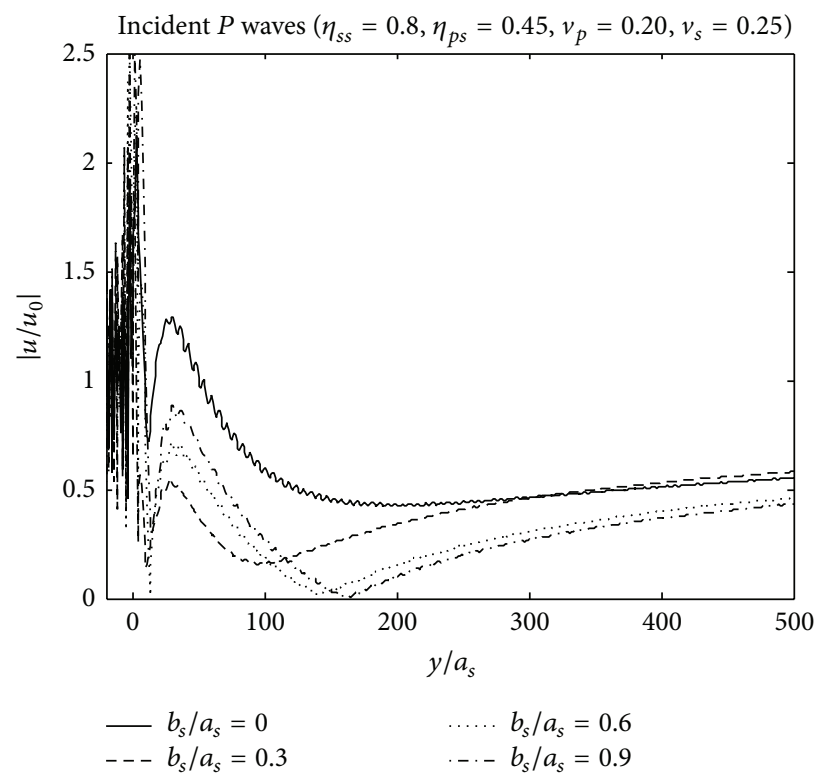

(d) $h=3.0 a_{s}$

Figure 5: Normalized displacement amplitudes curves along midline behind the isolation system under different ratios of internal and external diameters of tubular piles $\left(G_{r}=100, s_{p}=3.0 a_{s}, m=4, N=8\right)$.

distance $\left(y / a_{s}=150\right)$, whether SH or P waves incidence that is also a conformance resulted from Figure 3. $s_{p} / a_{s}=$ 3.0 is recommended as the designing criterion in practical engineering to ensure distinguished screening effects.

Another significant factor to measure the effectivity of barrier as isolation system is the so-called transmissibility index (TI) [10]:

$$
\begin{aligned}
& \operatorname{TI}_{u}\left(s_{p}, y\right)=\frac{1}{L} \int_{0}^{L}\left|\frac{u(x, y)}{u_{0}}\right| d x, \\
& \operatorname{TI}_{w}\left(s_{p}, y\right)=\frac{1}{L} \int_{0}^{L}\left|\frac{w(x, y)}{w_{0}}\right| d x,
\end{aligned}
$$

where $u$ and $w$ are defined as the total wave field, and those with subscripts 0 are demonstrated incident wave as well. Therefore, TI variesbetween 0 and 1 . The transmissibility indices with different positions $\left(100 \leq y / a_{s} \leq 200\right)$ distributed in the rear of barrier are illustrated by dash, dot dash, and solid line. The $x$-axis indicates that separations of tubular pile vary in the extent of $2.5 \leq s_{p} / a_{s} \leq 5.0$. As expected initially, despite carrying on rigid tubular piles $\left(G_{r}=1000\right)$, which are even equivalent with rigid solid piles, or elastic piles $\left(G_{r}=10\right)$, TI keeps a relatively lower range $\left(0.2 \leq \mathrm{TI}_{w} \leq 0.5,0.1 \leq \mathrm{TI}_{u} \leq 0.6\right)$ when pile spacing locates in certain limits $\left(2.5 \leq s_{p} / a_{s} \leq 3.5\right)$. It is 


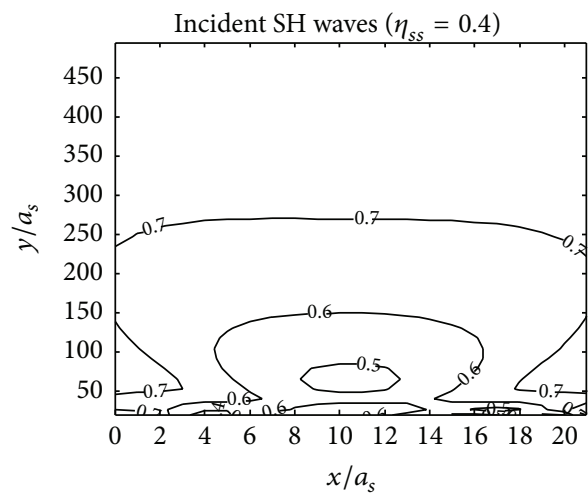

(a) $G_{r}=10$

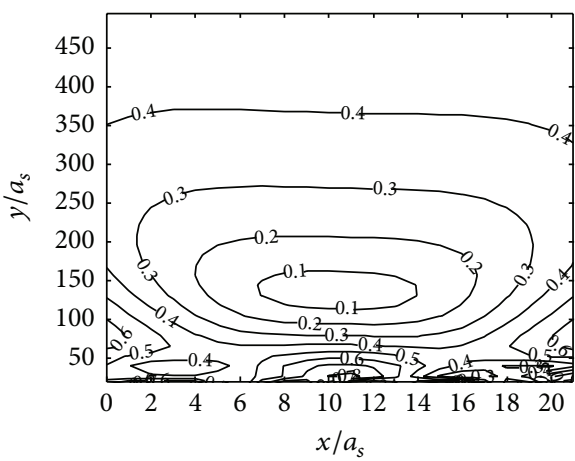

(c) $G_{r}=1000$

Incident $\mathrm{P}$ waves $\left(\eta_{s s}=0.8, \eta_{p s}=0.45\right.$,

$\left.v_{p}=0.20, v_{s}=0.25\right)$

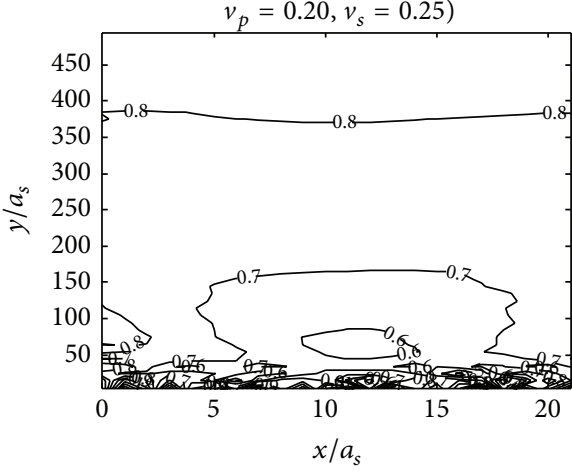

(e) $G_{r}=10$

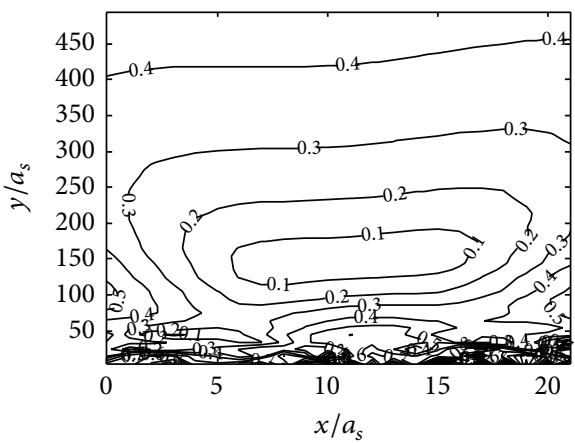

(g) $G_{r}=1000$

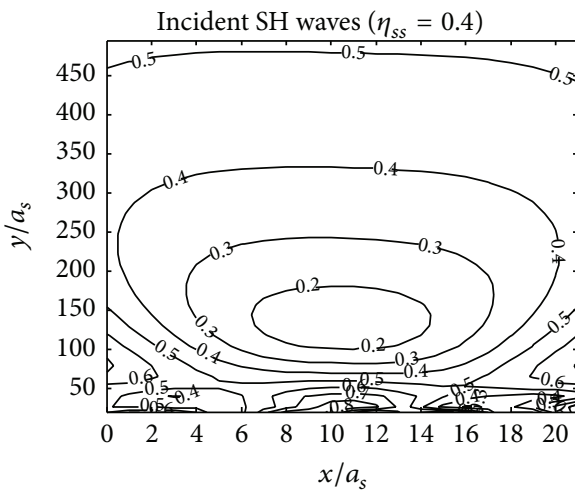

(b) $G_{r}=100$

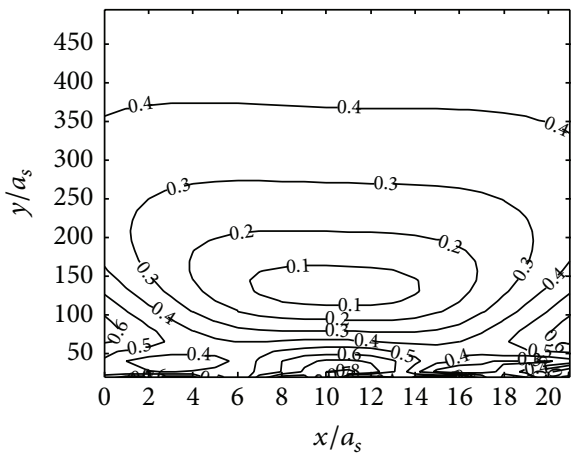

(d) $G_{r}=+\infty$

Incident $\mathrm{P}$ waves $\left(\eta_{s s}=0.8, \eta_{p s}=0.45\right.$,

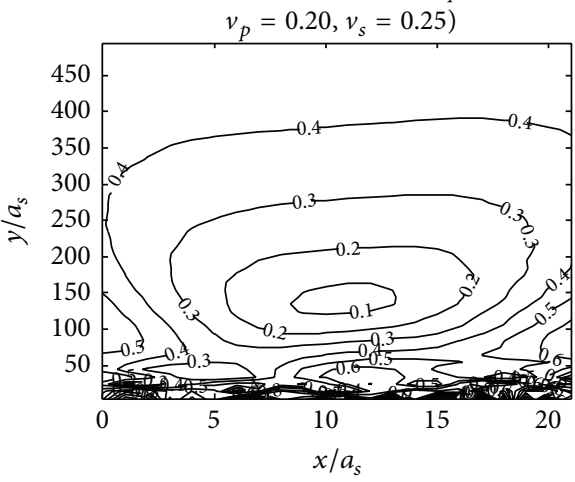

(f) $G_{r}=100$

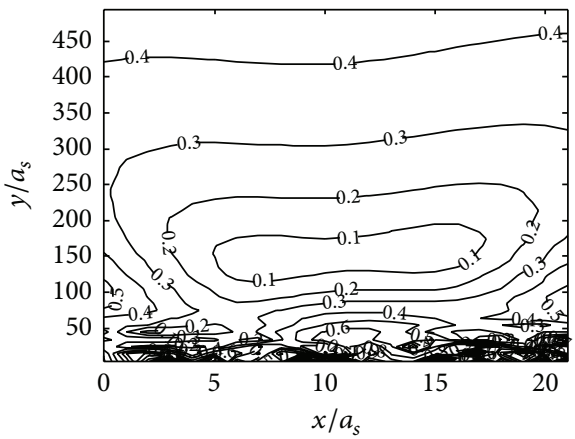

(h) $G_{r}=+\infty$

FIGURE 6: Contours of normalized displacement amplitudes by double-row tubular piles $\left(s_{p}=3.0 a_{s}, h=3.0 a_{s}, b_{s}=0.9 a_{s}, m=4, N=8\right)$. 


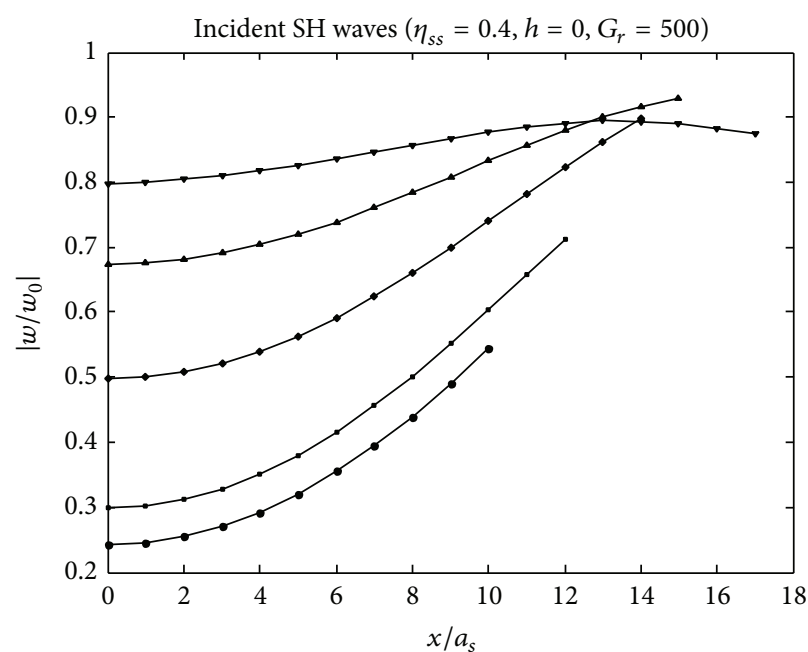

(a) $y / a_{s}=150$

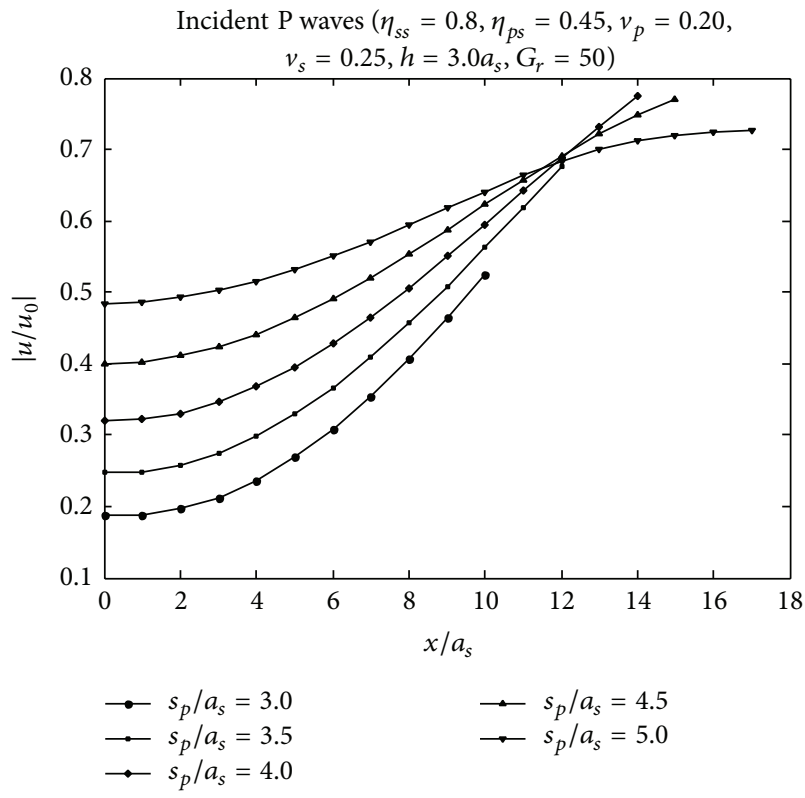

(c) $y / a_{s}=150$

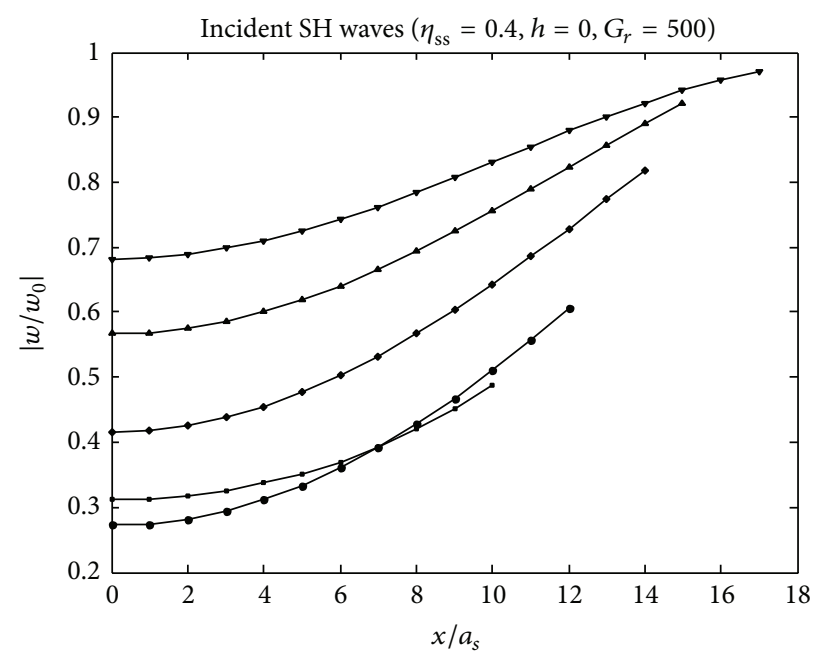

(b) $y / a_{s}=200$

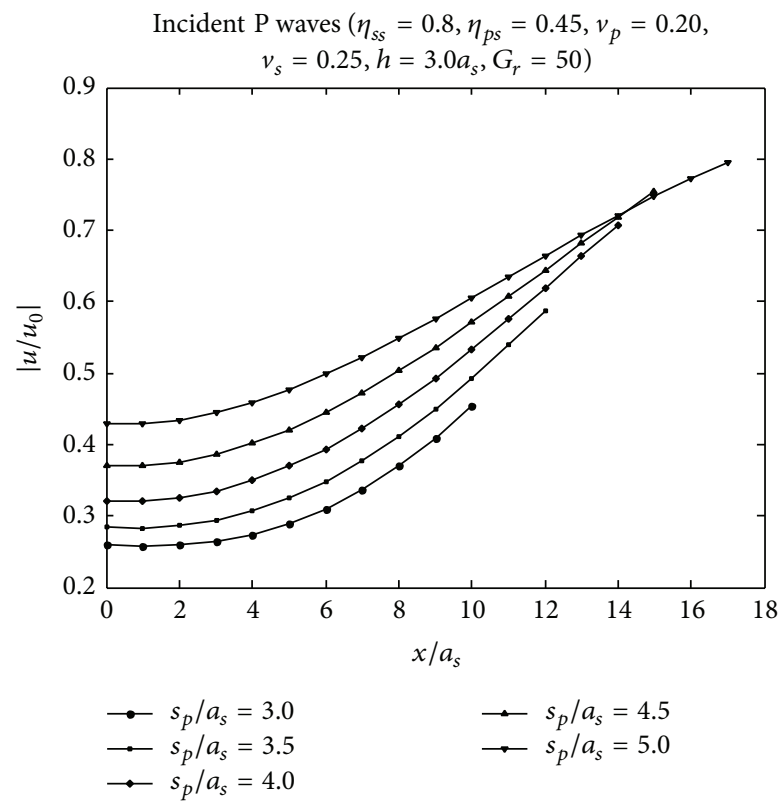

(d) $y / a_{s}=200$

FIGURE 7: Normalized displacement amplitudes curves with different separations of tubular piles $\left(b_{s}=0.9 a_{s}, m=4, N=8\right)$.

a proof that discontinuous barrier plays a role of integrity other than those expressed as disperse individuals. However, on the other side of view, Figures 8(c) and 8(d) $\left(G_{r}=1000\right)$ display a more preferable result in rigid tubular pile than under the circumstances of elastic ones in Figures 8(e) and 8(f) $\left(G_{r}=10\right)$, which is an affirmation with Section 3.4. Neither nearer $\left(y / a_{s}=100\right)$ nor farther $\left(y / a_{s}=250\right)$ confines could bring about an ideal screening effectiveness only when their positions of far field points are within $150 \leq y / a_{s} \leq 200$. Double-row tubular piles (Figures 8(c) and 8(d), $h=3.0 a_{s}$ ) evidently reveal outstripping isolating characteristics other than single-row (Figures 8(a) and $8(\mathrm{~b}), h=0$ ). Consequently, it is another vigorous identification that $2.5 \leq s_{p} / a_{s} \leq 3.5$ can be utilized as the most optimum spacing to ensure the desired effect of pile rows.

3.6. Influences of Distances $h$ between Tubular Pile Rows. The influence of multirow separation distance between tubular pile rows is investigated in Figure 9. Those figures of meshes represent intuitionistic isolation characters where the back of the discontinuous barrier is located. Aside from ubiquitous amplification of displacement amplitudes near the piles, one can perceive a sharp decrease presented in a certain range, almost shaping like low-lying hollows concentrated at the front. In the case of $h=0$ in Figure 9(a), the screening effectiveness performs as a single-row which $\left|w / w_{0}\right|$ keeps at 


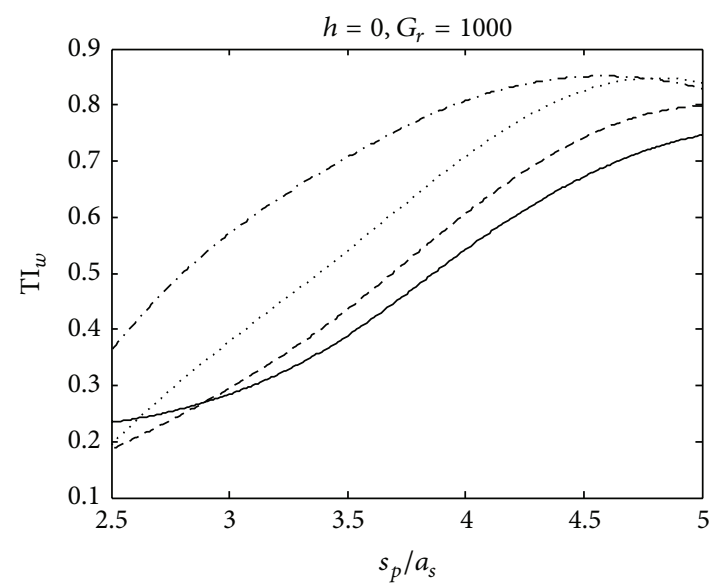

(a) Incident $\mathrm{SH}$ waves $\left(\eta_{s s}=0.4\right)$

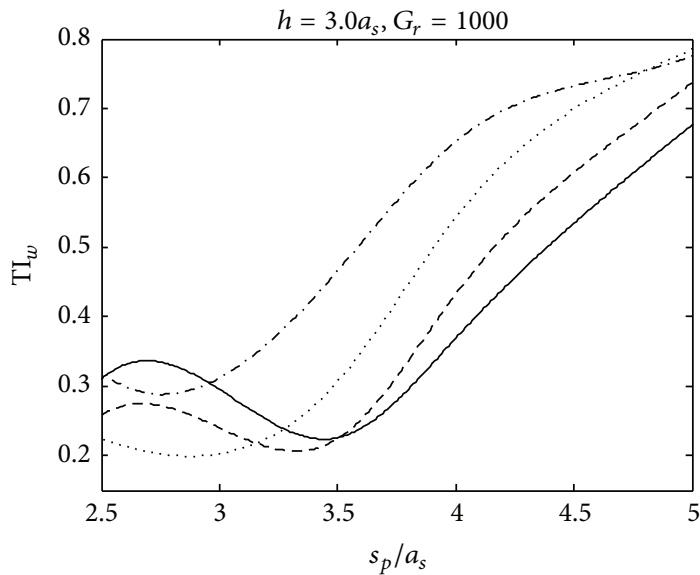

(c) Incident $\mathrm{SH}$ waves $\left(\eta_{s s}=0.4\right)$

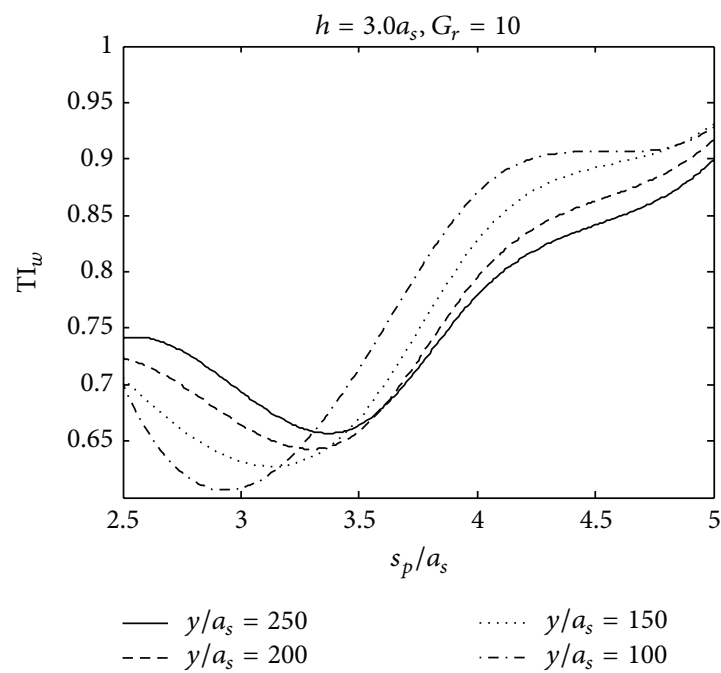

(e) Incident $\mathrm{SH}$ waves $\left(\eta_{s s}=0.4\right)$

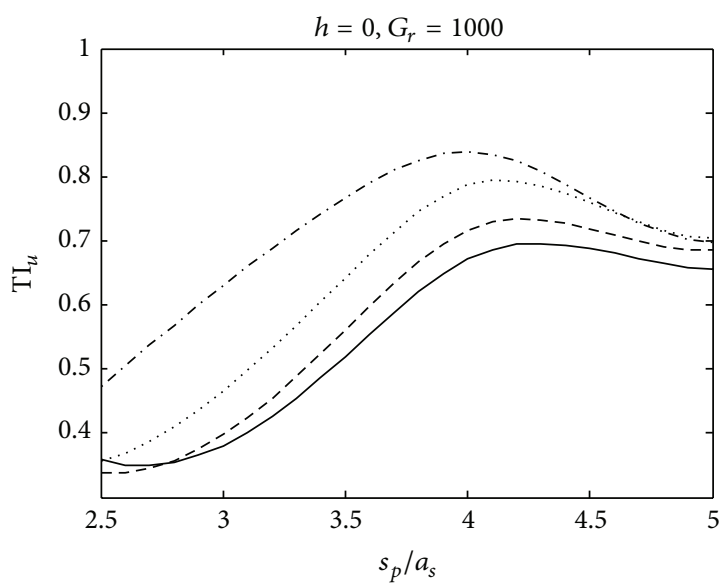

(b) Incident $\mathrm{P}$ waves $\left(\eta_{s s}=0.8, \eta_{p s}=0.45, v_{p}=0.20, v_{s}=\right.$ 0.25 )

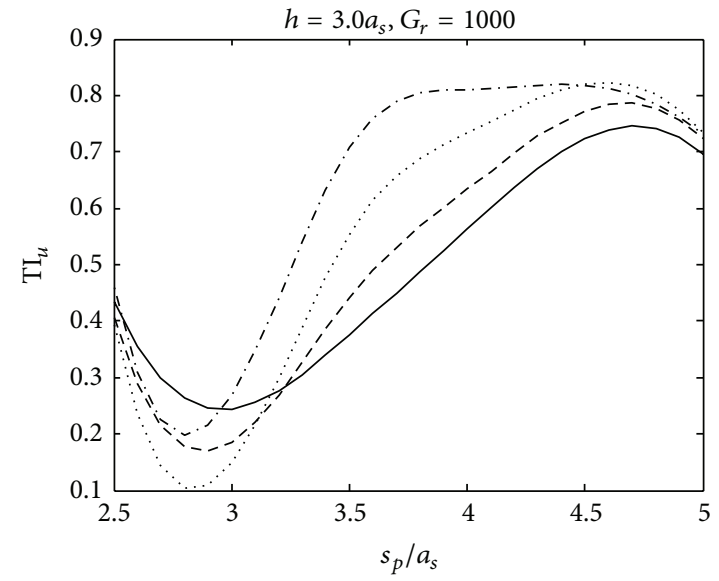

(d) Incident $\mathrm{P}$ waves $\left(\eta_{s s}=0.8, \eta_{p s}=0.45, v_{p}=0.20, v_{s}=\right.$ 0.25 )

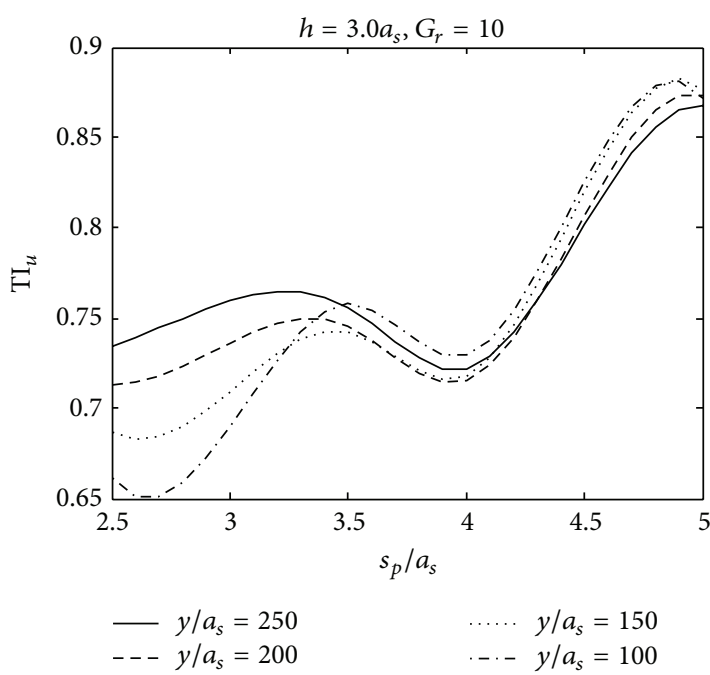

(f) Incident $\mathrm{P}$ waves $\left(\eta_{s s}=0.8, \eta_{p s}=0.45, v_{p}=0.20, v_{s}=\right.$ $0.25)$

FIGURE 8: Transmissibility index curves with different separations of tubular piles $\left(b_{s}=0.9 a_{s}, m=4, N=8\right)$. 


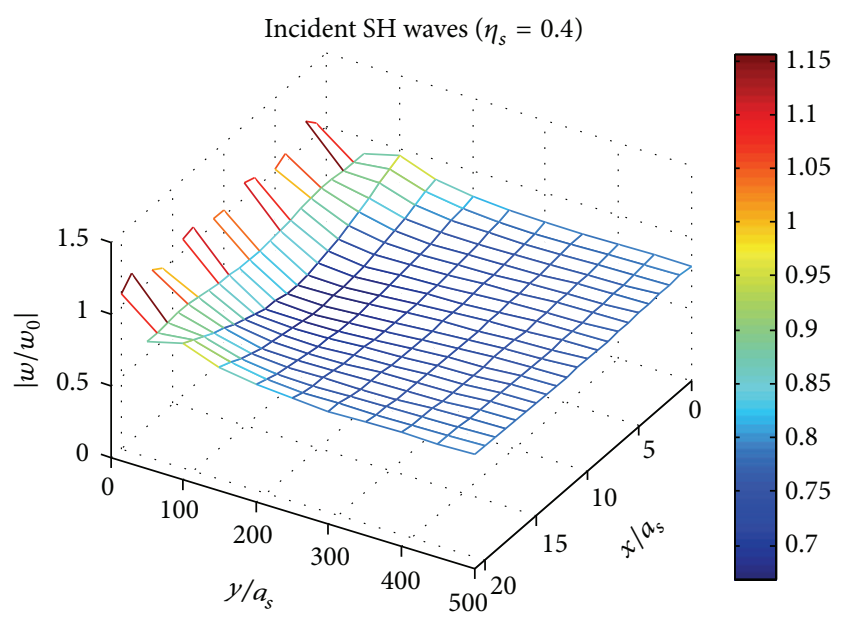

(a) $h=0, G_{r}=10$

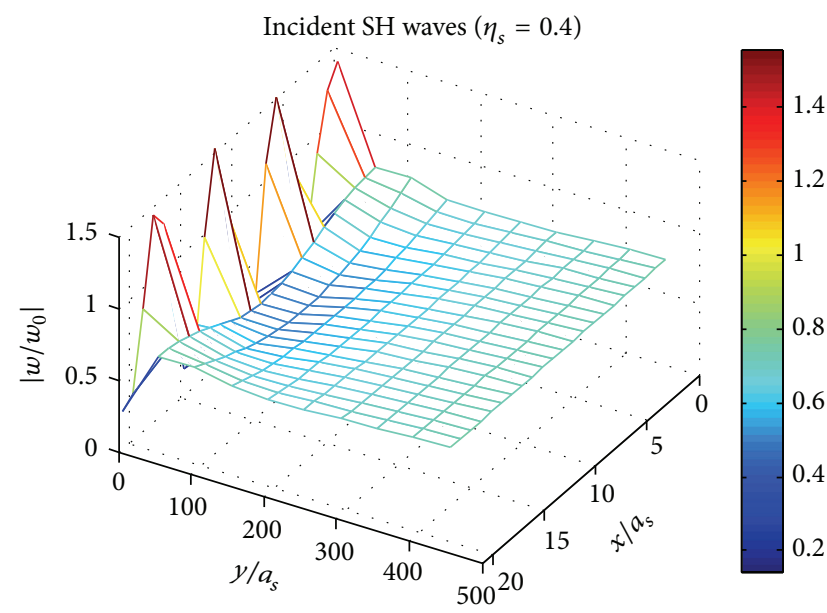

(c) $h=3.5 a_{s}, G_{r}=10$

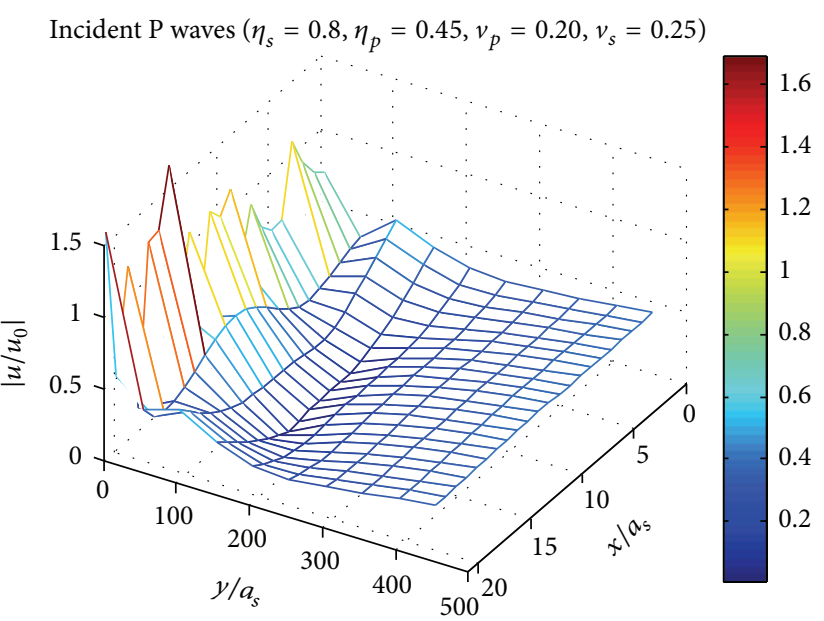

(b) $h=3.0 a_{s}, G_{r}=1000$

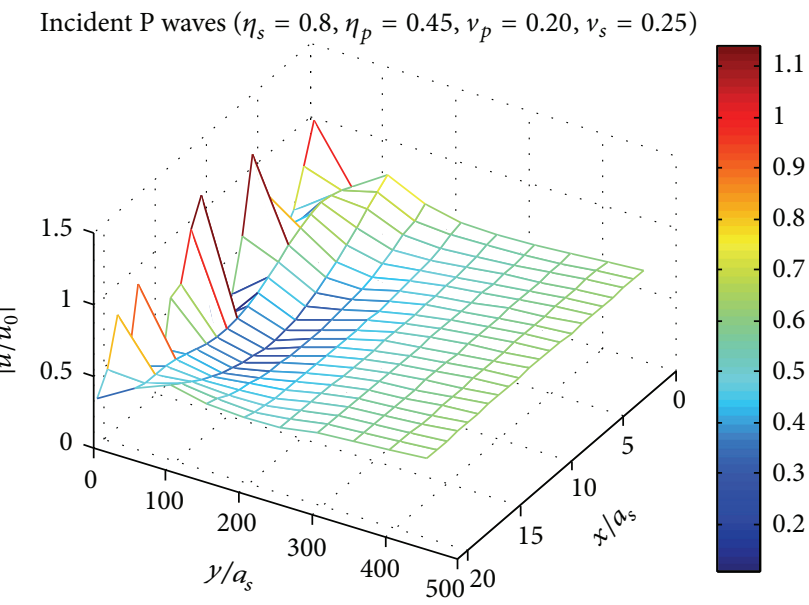

(d) $h=5.0 a_{s}, G_{r}=1000$

FIGURE 9: Normalized displacement amplitudes meshes behind the isolation system under different row-separations of tubular piles $\left(b_{s}=\right.$ $\left.0.9 a_{s}, s_{p}=3.0 a_{s}, m=4, N=8\right)$.

an average of $0.7 \sim 0.8$. With the larger separations between pile rows in Figure $9(\mathrm{c})\left(h=3.5 a_{s}\right)$ it takes, the $\left|w / w_{0}\right|$ reduces to $0.4 \sim 0.6$. It is a valid fact that suggests retaining particular spacing distances of tubular pile rows, a much more remarkable effect it will get without increasing the number of piles. Similar meshes appearance is shown in Figures 9(b) and 9(d) with $G_{r}=1000$ under the incidence of $\mathrm{P}$ waves. $\left|u / u_{0}\right|$ grows from $0.2 \sim 0.4$ to $0.6 \sim 0.7$ accompanied with $h$ rises. The increasing gap results in interaction phenomenon weakened so that the pile group no longer serves as a whole entirety. That is to say, not only pile separation but also row distance is the significant factor to form thickness of the obstacle to recede both incident $\mathrm{SH}$ and $\mathrm{P}$ waves. More prospective effectiveness on condition that multirow separation distance is $3.0 a_{s} \leq h \leq$ $3.5 a_{s}$ will be guaranteed.

3.7. Influences of Tubular Pile Numbers $N$. The numbers of piles varied from 6 to 12 along the midline behind pile rows are illustrated in Figure 10. In general, displacement amplitudes along the midline go to smaller when the numbers of piles increase. $\left|w / w_{0}\right|$ and $\left|u / u_{0}\right|$ damp at a more distant bound with more tubular piles. To some extent, a 12-pile barrier screens off $70-80 \%$ incident $\mathrm{P}$ waves while 6-pile barrier screens no more than $50-60 \%$ of it. Aside from the phenomena observed, an approximate result has been obtained in Figure 10(b), in which $G_{r}$ tending to a certain large value make the tubular pile coincide with rigid solid pile case. This degradation suggests a supportive fact of analytic solution of tubular pile from another point of view.

Numbers of rows, namely, the thickness of barrier, are an important factor affecting isolation of tubular piles. A treble-row shaped hexagon is illustrated in Figure 11, which displayed 12 piles versus 8 ones before. Comparing Figure 6(a) with Figure 12(a), it is observed that with growing of $50 \%$ pile numbers, which is double the thickness of screen, the minimum value $\left|w / w_{0}\right|$ of treble-row piles reduces to 0.1 . 


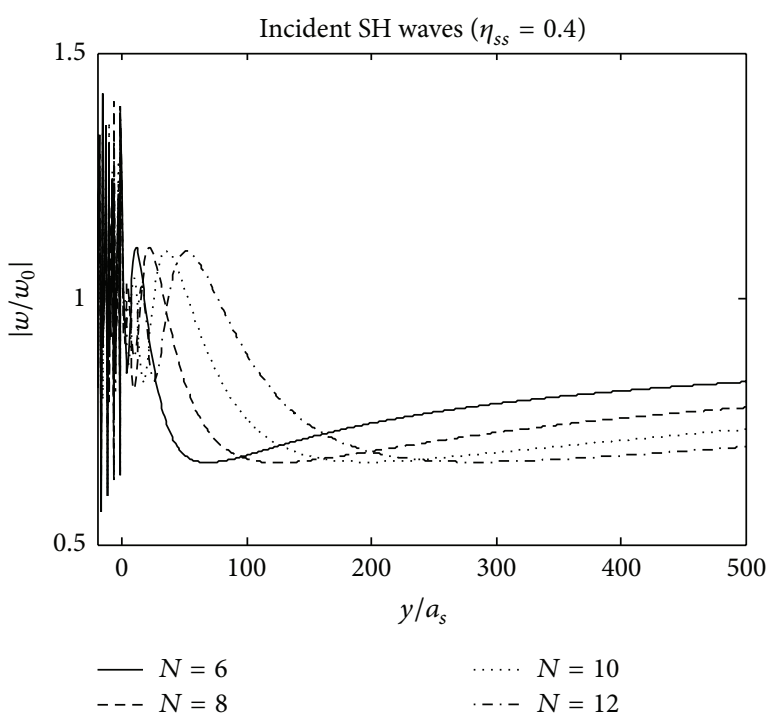

(a) $h=0, G_{r}=10$

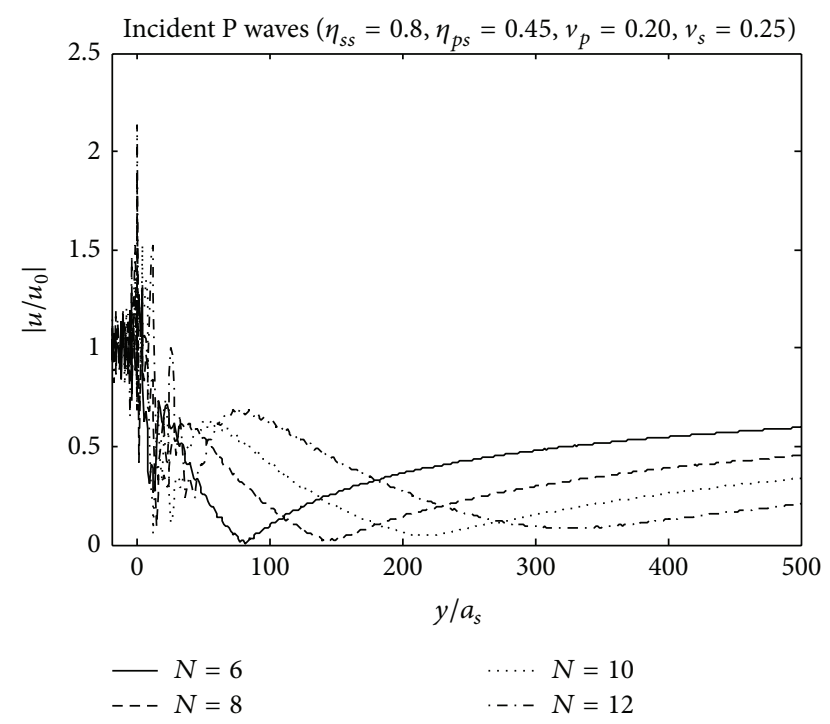

(b) $h=3 a_{s}, G_{r}=1000$

FIGURE 10: Normalized displacement amplitudes curves along midline behind the isolation system under different numbers of tubular piles $\left(b_{s}=0.9 a_{s}, s_{p}=3.0 a_{s}, m=4, N=8\right)$.

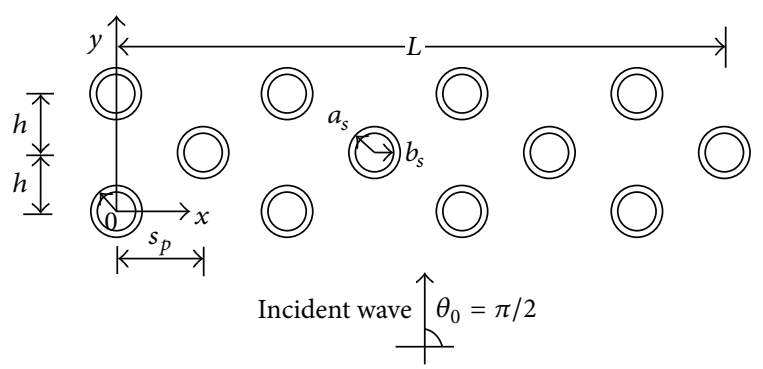

FIGURE 11: The reference system and analysis model of incident plane waves scattering by multirows of piles (hexagon arrangement, $N=$ 12).

Incident $\mathrm{SH}$ waves could been isolated no less than $40 \%$ at most area behind barrier. The minimum value $\left|w / w_{0}\right|$ of double-row is 0.5 at certain distance $\left(100 \leq y / a_{s} \leq 250\right)$ comparatively, and most of the region behind the screen incident waves is insulated only $30 \%$ at most region behind the screen. Analogous appearances can be received from Figure 6(e) and Figure 12(b) when it is of incident $\mathrm{P}$ waves.

3.8. Influences of Configuration of Tubular Piles. This paper presents a methodology of calculating scattering by arbitrarily arranged tubular piles. Different settings lead to diverse performances of isolation effect. Figure 13 shows the rectangular configuration reference system and Figure 14 depicts contours of normalized displacement amplitudes by incident $\mathrm{SH}$ and $\mathrm{P}$ waves. With the same numbers of tubular piles $(N=8)$, it can be perceived that no less than $40 \%$ of incident $\mathrm{SH}$ waves are screened when the barrier is hexagon shaped versus $20 \%$ of the incident waves isolated when the barrier is rectangular arranged from Figures 6(a) and 14(a), which increases double efficiency of isolation while it is hexagon. An obvious contrast can be discovered in Figures 14(b) and 6(e) under the incidence of $\mathrm{P}$ waves. Therefore, rectangular shaped system actually broadens the separations between the tubular piles with the same width of barrier so that less interaction and interference will occur. Reasonable arrangement is acceptable to meet the requirement of vibration attenuation in practical engineering.

\section{Conclusions}

A multiple scattering method different from the single scattering of calculating elastic waves scattering by an arbitrary configuration and radius of parallel cylindrical tubular piles is proposed. By means of constructing transmitted waves field inside the tubular piles, applying elastic pile-soil interface boundary conditions, and employing wave function expansion and coordinates conversion theory, it acquires scattering and transmitted complex coefficients of each order. Total scattering and transmitted wave fields are determined consequently. Example analysis of single and multirow tubular piles, which are focused on scattering orders, types of incident waves, materials of screen, and geometrical parameters have been discussed.

(1) An amplification phenomenon of displacement amplitudes behind the tubular pile screen stimulated by the incident plane waves is conspicuous. Displacement amplitudes increment appears to be gradually decreasing with the orders of scattering increasing ( $m=3$ and 4 ) and is nearly superposed far apart the barrier $\left(y / a_{s} \geq 400\right)$ especially. It is concerned that displacement amplitudes are 


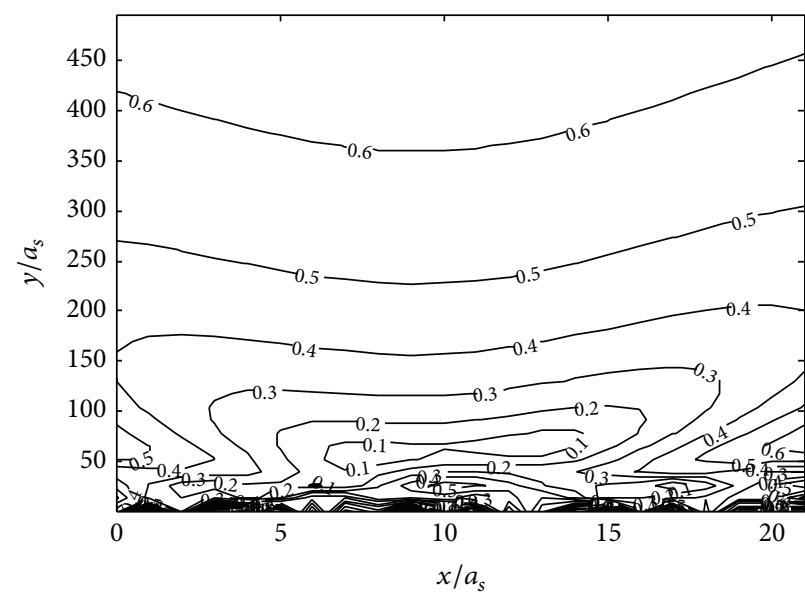

(a) Incident $\mathrm{SH}$ waves $\left(\eta_{s s}=0.4\right)$

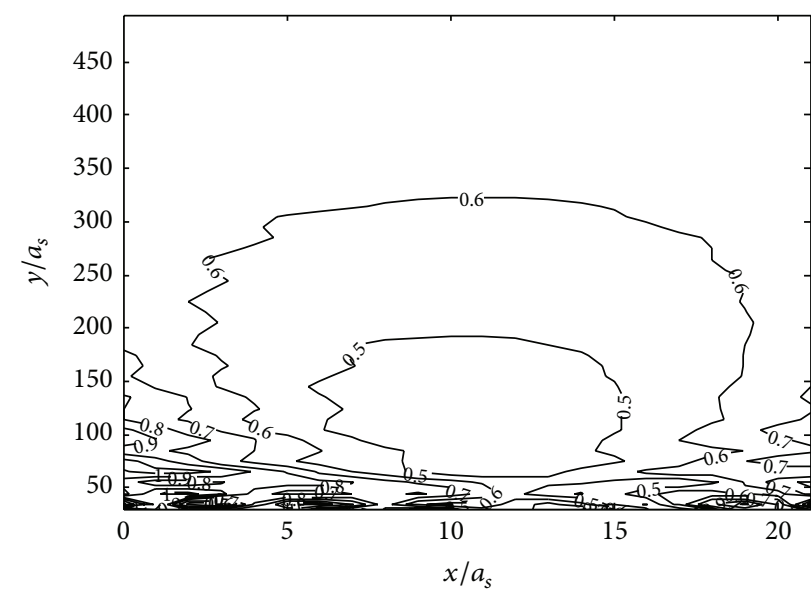

(b) Incident $\mathrm{P}$ waves $\left(\eta_{s s}=0.8, \eta_{p s}=0.45, v_{p}=0.20, v_{s}=0.25\right)$

FIGURE 12: Contours of normalized displacement amplitudes by multirows of tubular piles $\left(b_{s}=0.9 a_{s}, s_{p}=3.0 a_{s}, h=3.0 a_{s}, G_{r}=10, m=\right.$ $4, N=12$ ).

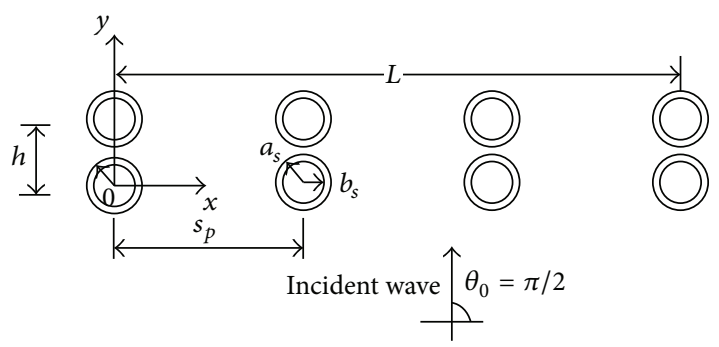

FIGURE 13: The reference system and analysis model of incident plane waves scattering by multirows of tubular piles (rectangular arrangement, $N=8$ ).

converged when $m$ truncates at 3 to 4 . No matter what kind of incident waves, the computation of multiple scattering method is much smaller than the way based on single scattering hypothesis. It is presented to be the basis of pile barrier optimal design in practical engineering.

(2) The most prominent amplification of displacement is discovered centered behind the pile barrier. Meanwhile, the minimum values of that appear exactly behind the amplification zone and the amplitudes of two sides are apparently varying small. It indicates that a similar manifestation like continuous barrier characteristics is achieved by tubular piles.

(3) Internal and external radius ratio or called thickness of tube wall is a significant influencing factor of screening effectiveness which is distinct from solid piles. Whether single or multiple rows of them, a reduction of thickness makes displacement amplitudes weakened remarkably. When internal radius $b_{s}$ approaches to 0 , that is, degenerating to elastic solid piles, the decrement of displacement is less than that of tubular piles of the same external radius. As a result, tubular pile is an ideal shape of much beneficial and practical substitute of discontinuous barrier for solid ones. $b_{s} / a_{s}=0.9$ is proved to be the optimum ratio to acquire better isolation result.

(4) The shear modulus ratio of materials of piles and soil, or say the stiffness of barrier, influences the function of screening directly. An obvious magnification emerges with the pile-soil shear modulus ratio increasing; the optimum isolation region falls back and the minimum displacement amplitudes decrease so that the isolation effectiveness improves evidently. In addition, tubular pile groups with $G_{r}=1000$ are regarded as fixed rigid piles for the normalized displacement amplitudes contour is tantamount to that of $G_{r}=+\infty$.

(5) Variation of separation between piles has much impact on screening of incident waves. It is more sensitive to displacement increment at central area than at two sides behind the barrier. TI which is called transmitted index is introduced to evaluate the isolation efficiency. The transmitted waves become less when the separations of single-row piles and double-row reduce. The best separation of tubular piles tends to be $2.5 \leq s_{p} / a_{s} \leq 3.5$.

(6) Row-to-row distance needs to keep certain range because pile rows are isolated as discrete barrier gradually when the distance increases. With the same numbers of tubular piles, double-row performs more superiorly than single-row tubular piles. It works sufficiently when distance is about $3.0 a_{s} \leq h \leq 3.5 a_{s}$.

(7) Increasing either of width or thickness of barrier gives rise to improvement of the effectiveness of screening. The rectangle configuration widens the separation of tubular piles and reduces the chances of multiple scattering coherent phases' intervention. 


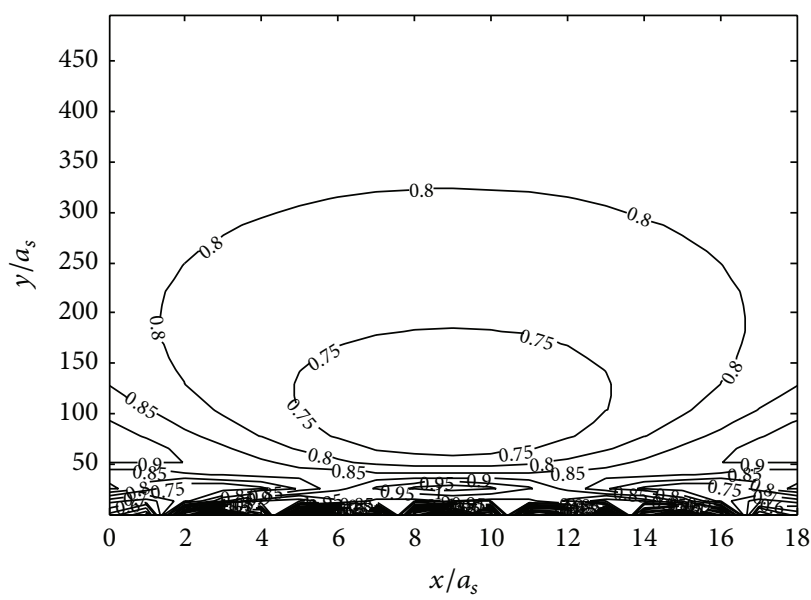

(a) Incident SH waves $\left(\eta_{s s}=0.4\right)$

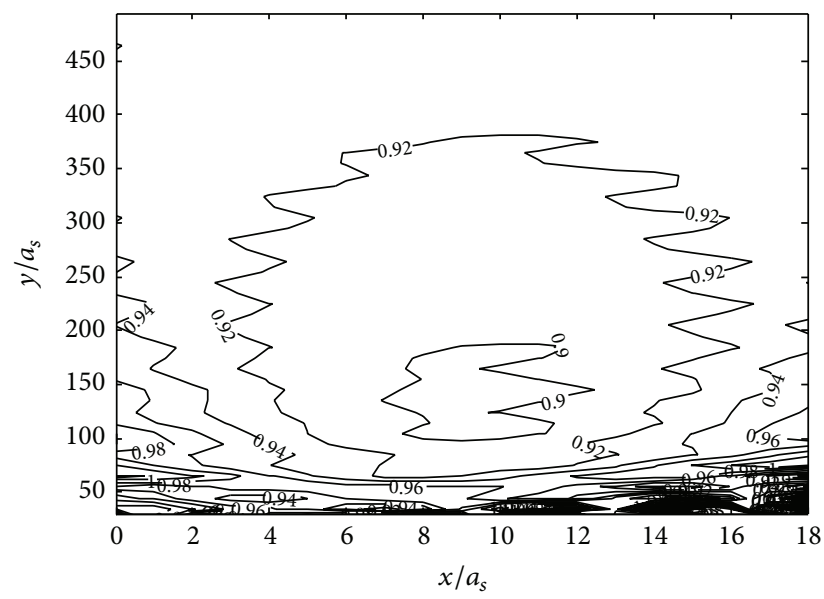

(b) Incident $\mathrm{P}$ waves $\left(\eta_{s s}=0.8, \eta_{p s}=0.45, v_{p}=0.20, v_{s}=0.25\right)$

FIGURE 14: Contours of normalized displacement amplitudes by double-rows of tubular piles (rectangular arrangement, $b_{s}=0.9 a_{s}, s_{p}=6.0 a_{s}$, $\left.h=3.0 a_{s}, G_{r}=10, m=4, N=8\right)$.

Compared with rectangle arrangement, hexagon configuration with the same numbers of tubular piles is preferred.

\section{Appendix}

Elements of matrices are

$$
\begin{aligned}
& p_{11}=-\alpha_{s} \mathrm{H}_{n}^{\prime(1)}\left(\alpha_{s} a_{s}\right), \quad p_{12}=\frac{n}{a_{s}} \mathrm{H}_{n}^{(1)}\left(\beta_{s} a_{s}\right), \\
& p_{13}=\alpha_{p} \mathrm{H}_{n}^{\prime(1)}\left(\alpha_{p} a_{s}\right), \quad p_{14}=\alpha_{p} \mathrm{H}_{n}^{\prime(2)}\left(\alpha_{p} a_{s}\right) \text {, } \\
& p_{15}=-\frac{n}{a_{s}} \mathrm{H}_{n}^{(1)}\left(\beta_{p} a_{s}\right), \quad p_{16}=-\frac{n}{a_{s}} \mathrm{H}_{n}^{(2)}\left(\beta_{p} a_{s}\right) \text {; } \\
& p_{21}=-\frac{n}{a_{s}} \mathrm{H}_{n}^{(1)}\left(\alpha_{s} a_{s}\right), \quad p_{22}=\beta_{s} \mathrm{H}_{n}^{\prime(1)}\left(\beta_{s} a_{s}\right), \\
& p_{23}=\frac{n}{a_{s}} \mathrm{H}_{n}^{(1)}\left(\alpha_{p} a_{s}\right), \quad p_{24}=\frac{n}{a_{s}} \mathrm{H}_{n}^{(2)}\left(\alpha_{p} a_{s}\right), \\
& p_{25}=-\beta_{p} \mathrm{H}_{n}^{\prime(1)}\left(\beta_{p} a_{s}\right), \quad p_{26}=-\beta_{p} \mathrm{H}_{n}^{\prime(2)}\left(\beta_{p} a_{s}\right) \text {; } \\
& p_{31}=-\frac{1}{G_{r}} \mathscr{E}_{11}^{(3)}\left(\alpha_{s} a_{s}\right), \quad p_{32}=-\frac{1}{G_{r}} \mathscr{E}_{12}^{(3)}\left(\beta_{s} a_{s}\right) \text {, } \\
& p_{33}=\mathscr{E}_{11}^{(3)}\left(\alpha_{p} a_{s}\right), \quad p_{34}=\mathscr{E}_{11}^{(4)}\left(\alpha_{p} a_{s}\right), \\
& p_{35}=\mathscr{E}_{12}^{(3)}\left(\beta_{p} a_{s}\right), \quad p_{36}=\mathscr{E}_{12}^{(4)}\left(\beta_{p} a_{s}\right) ; \\
& p_{41}=-\frac{1}{G_{r}} \mathscr{E}_{41}^{(3)}\left(\alpha_{s} a_{s}\right) \quad p_{42}=-\frac{1}{G_{r}} \mathscr{E}_{42}^{(3)}\left(\beta_{s} a_{s}\right) \text {, } \\
& p_{43}=\mathscr{E}_{41}^{(3)}\left(\alpha_{p} a_{s}\right), \quad p_{44}=\mathscr{E}_{41}^{(4)}\left(\alpha_{p} a_{s}\right), \\
& p_{45}=\mathscr{E}_{42}^{(3)}\left(\beta_{p} a_{s}\right), \quad p_{46}=\mathscr{E}_{42}^{(4)}\left(\beta_{p} a_{s}\right) ; \\
& p_{51}=0, \quad p_{52}=0, \quad p_{53}=\mathscr{E}_{11}^{(3)}\left(\alpha_{p} b_{s}\right) \text {, } \\
& p_{54}=\mathscr{E}_{11}^{(4)}\left(\alpha_{p} b_{s}\right), \quad p_{55}=\mathscr{E}_{12}^{(3)}\left(\beta_{p} b_{s}\right) \text {, } \\
& p_{56}=\mathscr{E}_{12}^{(4)}\left(\beta_{p} b_{s}\right) \text {; }
\end{aligned}
$$

$$
\begin{aligned}
& p_{61}=0, \quad p_{62}=0, \\
& p_{63}=\mathscr{E}_{41}^{(3)}\left(\alpha_{p} b_{s}\right), \quad p_{64}=\mathscr{E}_{41}^{(4)}\left(\alpha_{p} b_{s}\right), \\
& p_{65}=\mathscr{E}_{42}^{(3)}\left(\beta_{p} b_{s}\right), \quad p_{66}=\mathscr{E}_{42}^{(4)}\left(\beta_{p} b_{s}\right) ; \\
& q_{1}=\alpha_{s} \mathrm{~J}_{n}^{\prime}\left(\alpha_{s} a_{s}\right)^{s} \theta_{\alpha}, \quad q_{2}=\frac{n}{a_{s}} \mathrm{~J}_{n}\left(\alpha_{s} a_{s}\right)^{s} \theta_{\alpha}, \\
& q_{3}=\frac{1}{G_{r}} \mathscr{E}_{11}^{(1)}\left(\alpha_{s} a_{s}\right)^{s} \theta_{\alpha}, \quad q_{4}=\frac{1}{G_{r}} \mathscr{E}_{41}^{(1)}\left(\alpha_{s} a_{s}\right)^{s} \theta_{\alpha}, \\
& q_{5}=0, \quad q_{6}=0 \\
& r_{11}=\sum_{s^{\prime}=0, s^{\prime} \neq s}^{N^{\prime}} \sum_{n^{\prime}=-\infty}^{+\infty} \alpha_{s} J_{n}^{\prime}\left(\alpha_{s} a_{s}\right) s \mathscr{H}_{n^{\prime} n}\left(\alpha_{s} a_{s s^{\prime}}\right) \text {, } \\
& r_{12}=-\sum_{s^{\prime}=0, s^{\prime} \neq s}^{N^{\prime}} \sum_{n^{\prime}=-\infty}^{+\infty} \frac{n^{\prime}}{a_{s^{\prime}}}{ }^{s} \mathrm{~J}_{n}\left(\beta_{s} a_{s}\right) \mathscr{H}_{n^{\prime} n}\left(\beta_{s} a_{s s^{\prime}}\right) \text {, } \\
& r_{21}=\sum_{s^{\prime}=0, s^{\prime} \neq s}^{N^{\prime}} \sum_{n^{\prime}=-\infty}^{+\infty} \frac{n^{\prime}}{a_{s^{\prime}}} J_{n}\left(\alpha_{s} a_{s}\right){ }^{s} \mathscr{H}_{n^{\prime} n}\left(\alpha_{s} a_{s s^{\prime}}\right) \text {, } \\
& r_{22}=-\sum_{s^{\prime}=0, s^{\prime} \neq s}^{N^{\prime}} \sum_{n^{\prime}=-\infty}^{+\infty} \beta_{s} J_{n}^{\prime}\left(\beta_{s} a_{s}\right){ }^{s} \mathscr{H}_{n^{\prime} n}\left(\beta_{s} a_{s s^{\prime}}\right) \text {, } \\
& r_{31}=\sum_{s^{\prime}=0, s^{\prime} \neq s}^{N^{\prime}} \sum_{n^{\prime}=-\infty}^{+\infty} \frac{a_{s}^{2}}{G_{r} a_{s^{\prime}}^{2}} \mathscr{E}_{11}^{(1)}\left(\alpha_{s} a_{s}\right) s \mathscr{H}_{n^{\prime} n}\left(\alpha_{s} a_{s s^{\prime}}\right) \text {, } \\
& r_{32}=\sum_{s^{\prime}=0, s^{\prime} \neq s}^{N^{\prime}} \sum_{n^{\prime}=-\infty}^{+\infty} \frac{a_{s}^{2}}{G_{r} a_{s^{\prime}}^{2}} \mathscr{E}_{12}^{(1)}\left(\beta_{s} a_{s}\right){ }^{s} \mathscr{H}_{n^{\prime} n}\left(\beta_{s} a_{s s^{\prime}}\right) \text {, } \\
& r_{41}=\sum_{s^{\prime}=0, s^{\prime} \neq s}^{N^{\prime}} \sum_{n^{\prime}=-\infty}^{+\infty} \frac{a_{s}^{2}}{G_{r} a_{s^{\prime}}^{2}} \mathscr{E}_{41}^{(1)}\left(\alpha_{s} a_{s}\right){ }^{s} \mathscr{H}_{n^{\prime} n}\left(\alpha_{s} a_{s s^{\prime}}\right) \text {, }
\end{aligned}
$$




$$
\begin{gathered}
r_{42}=\sum_{s^{\prime}=0, s^{\prime} \neq s}^{N^{\prime}} \sum_{n^{\prime}=-\infty}^{+\infty} \frac{a_{s}^{2}}{G_{r} a_{s^{\prime}}^{2}} \mathscr{E}_{42}^{(1)}\left(\beta_{s} a_{s}\right){ }^{s} \mathscr{H}_{n^{\prime} n}\left(\beta_{s} a_{s s^{\prime}}\right), \\
r_{51}=0, \quad r_{52}=0, \quad r_{61}=0, \quad r_{62}=0 ; \\
s_{1}=\mathrm{i} \frac{n}{a_{s}} \mathrm{~J}_{n}\left(\beta_{s} a_{s}\right){ }^{s} \theta_{\beta}, \\
s_{2}=-\mathrm{i} \beta_{s} \mathrm{~J}_{n}^{\prime}\left(\beta_{s} a_{s}\right){ }^{s} \theta_{\beta}, \quad s_{3}=\mathrm{i} \frac{1}{G_{r}} \mathscr{E}_{41}^{(1)}\left(\beta_{s} a_{s}\right){ }^{s} \theta_{\beta}, \\
s_{4}=-\mathrm{i} \frac{1}{G_{r}} \mathscr{E}_{11}^{(1)}\left(\alpha_{s} a_{s}\right){ }^{s} \theta_{\beta}, \quad s_{5}=0, \quad s_{6}=0,
\end{gathered}
$$

where

$$
\begin{aligned}
& \mathscr{E}_{11}^{(1)}\left(\alpha r_{s}\right)=-2\left(\frac{\beta r_{s}}{2}\right)^{2} \mathrm{~J}_{n}\left(\alpha r_{s}\right)+\left(\frac{\alpha r_{s}}{2}\right)^{2} \\
& \cdot\left[\mathrm{J}_{n+2}\left(\alpha r_{s}\right)+2 \mathrm{~J}_{n}\left(\alpha r_{s}\right)+\mathrm{J}_{n-2}\left(\alpha r_{s}\right)\right], \\
& \mathscr{E}_{11}^{(3)}\left(\alpha r_{s}\right)=-2\left(\frac{\beta r_{s}}{2}\right)^{2} \mathrm{H}_{n}\left(\alpha r_{s}\right)+\left(\frac{\alpha r_{s}}{2}\right)^{2} \\
& \cdot\left[\mathrm{H}_{n+2}\left(\alpha r_{s}\right)+2 \mathrm{H}_{n}\left(\alpha r_{s}\right)+\mathrm{H}_{n-2}\left(\alpha r_{s}\right)\right], \\
& \mathscr{E}_{12}^{(1)}\left(\beta r_{s}\right)=\left(\frac{\beta r_{s}}{2}\right)^{2}\left[\mathrm{~J}_{n+2}\left(\beta r_{s}\right)-\mathrm{J}_{n-2}\left(\beta r_{s}\right)\right], \\
& \mathscr{E}_{12}^{(3)}\left(\beta r_{s}\right)=\left(\frac{\beta r_{s}}{2}\right)^{2}\left[\mathrm{H}_{n+2}\left(\beta r_{s}\right)-\mathrm{H}_{n-2}\left(\beta r_{s}\right)\right], \\
& \mathscr{E}_{21}^{(3)}\left(\alpha r_{s}\right)= {\left[-\left(n^{2}+n\right)+\left(\alpha r_{s}\right)^{2}\right] } \\
& \times \mathrm{H}_{n}^{(1)}\left(\alpha r_{s}\right)+\left(\alpha r_{s}\right) \mathrm{H}_{n-1}^{(1)}\left(\alpha r_{s}\right), \\
& \mathscr{E}_{42}^{(1)}\left(\beta r_{s}\right)=\left(\frac{\beta r_{s}}{2}\right)^{2}\left[\mathrm{H}_{n+2}\left(\beta r_{s}\right)+\mathrm{H}_{n-2}\left(\beta r_{s}\right)\right], \\
& \mathscr{E}_{22}^{(4)}\left(\beta r_{s}\right)=\left(\frac{\beta r_{s}}{2}\right)^{2}\left[\mathrm{H}_{n-2}^{(2)}\left(\beta r_{s}\right)-\mathrm{H}_{n+2}^{(2)}\left(\beta r_{s}\right)\right], \\
& \mathscr{E}_{41}^{(1)}\left(\alpha r_{s}\right)=\left(\frac{\alpha r_{s}}{2}\right)^{2}\left[\mathrm{~J}_{n+2}\left(\alpha r_{s}\right)-\mathrm{J}_{n-2}\left(\alpha r_{s}\right)\right], \\
& \mathscr{E}_{22}^{(3)}\left(\beta r_{s}\right)=\left(\frac{\beta r_{s}}{2}\right)^{2}\left[\mathrm{H}_{n-2}\left(\beta r_{s}\right)-\mathrm{H}_{n+2}\left(\beta r_{s}\right)\right], \\
& \mathscr{E}_{21}^{(4)}\left(\alpha r_{s}\right)= {\left[-\left(n^{2}+n\right)+\left(\alpha r_{s}\right)^{2}\right] }
\end{aligned}
$$

in which the subscripts of $\alpha$ and $\beta$ can be $s$ or $p$.

\section{Conflict of Interests}

The authors declare that there is no conflict of interests regarding the publication of this paper.

\section{Acknowledgments}

The authors are grateful to the anonymous reviewers for their helpful advice and valuable comments. This work was supported by the National Natural Science Foundation of China under Grant (no. 51309201), Public Welfare Scientific Research Foundation of the Ministry of Water Resource of China (no. 201201043), and Post-doctoral Merited Research Foundation in Zhejiang Province of China (no. Bsh1202040).

\section{References}

[1] D. D. Barkan, Dynamics of Bases and Foundation, McGraw-Hill, New York, NY, USA, 1962.

[2] F. E. Richart, J. R. Hall, and R. D. Woods, Vibrations of Soils and Foundations, Prentice-Hall, Englewood Cliffs, NJ, USA, 1970.

[3] R. D. Woods, N. E. Barnett, and R. Sagesser, "Holography, a new tool for soil dynamics," Journal of Geotechnical Engineering, vol. 100, no. 11, pp. 1231-1247, 1974.

[4] W. A. Haupt, "Model test on screening of surface waves," in Proceedings of the 10th International Conference on Soil Mechanics and Foundation Engineering, vol. 3, pp. 215-222, Stockholm, Sweden, 1981.

[5] S. Liao and D. A. Sangrey, "Use of piles as isolation barriers," Journal of the Geotechnical Engineering Division, vol. 104, no. 9, pp. 1139-1152, 1978.

[6] B. Boroomand and A. M. Kaynia, "Stiffness and damping of closely spaced pile groups," in Proceedings of the 1st International Conference on Soil Dynamics and Earthquake Engineering, pp. 490-501, Karlsruhe, Germany, September 1991.

[7] Y. Kani and K. Hayakawa, "Simulation analysis about effects of a PC wall-pile barrier on reducing ground vibration," in Proceedings of the 13th International Offshore and Polar Engineering Conference, pp. 677-682, Honolulu, Hawaii, USA, 2003.

[8] S. E. Kattis, D. Polyzos, and D. E. Beskos, "Vibration isolation by a row of piles using a 3-D frequency domain BEM," International Journal for Numerical Methods in Engineering, vol. 46, no. 5, pp. 713-728, 1999.

[9] S. E. Kattis, D. Polyzos, and D. E. Beskos, "Modelling of pile wave barriers by effective trenches and their screening effectiveness," Soil Dynamics and Earthquake Engineering, vol. 18, no. 1, pp. 110, 1999.

[10] J. Aviles and F. J. Sanchez-Sesma, "Piles as barriers for elastic waves," Journal of Geotechnical Engineering, vol. 109, no. 9, pp. 1133-1146, 1983.

[11] J. Avilés and F. J. Sánchez-Sesma, "Foundation isolation from vibration using piles as barriers," Journal of Engineering Mechanics, vol. 114, no. 11, pp. 1854-1870, 1988.

[12] G. Y. Gao, Z. Y. Li, C. Qiu, and Z. Q. Yue, “Three-dimensional analysis of rows of piles as passive barriers for ground vibration isolation," Soil Dynamics and Earthquake Engineering, vol. 26, no. 11, pp. 1015-1027, 2006.

[13] Z.-Y. Li, G.-Y. Gao, C. Qiu, and X.-J. Yang, "Analysis of multirow of piles as barriers for isolating vibration in far field," Chinese Journal of Rock Mechanics and Engineering, vol. 24, no. 21, pp. 3990-3995, 2005. 
[14] P. Xu, T.-D. Xia, and X.-M. Zhou, "Study on effect of barrier of a row of hollow pipe piles on isolation of incident plane SV waves," Chinese Journal of Geotechnical Engineering, vol. 29, no. 1, pp. 131-136, 2007.

[15] P. Xu, X.-M. Zhou, and T.-D. Xia, "Discontinuous barrier used a row of elastic piles for incident elastic waves," Journal of Vibration Engineering, vol. 20, no. 4, pp. 388-395, 2007.

[16] Y.-Q. Cai, G.-Y. Ding, and C.-J. Xu, "Screening of plane S waves by an array of rigid piles in poroelastic soil," Journal of Zhejiang University: Science A, vol. 9, no. 5, pp. 589-599, 2008.

[17] Y.-Q. Cai, G.-Y. Ding, and C.-J. Xu, "Amplitude reduction of elastic waves by a row of piles in poroelastic soil," Computers and Geotechnics, vol. 36, no. 3, pp. 463-473, 2009.

[18] Y.-Q. Cai, G.-Y. Ding, C.-J. Xu, and J. Wang, "Vertical amplitude reduction of Rayleigh waves by a row of piles in a poroelastic half-space," International Journal for Numerical and Analytical Methods in Geomechanics, vol. 33, no. 16, pp. 1799-1821, 2009.

[19] P.-H. Tsai, Z.-Y. Feng, and T.-L. Jen, "Three-dimensional analysis of the screening effectiveness of hollow pile barriers for foundation-induced vertical vibration," Computers and Geotechnics, vol. 35, no. 3, pp. 489-499, 2008.

[20] T.-D. Xia, M.-M. Sun, C. Chen, W.-Y. Chen, and X. Ping, "Analysis on multiple scattering by an arbitrary configuration of piles as barriers for vibration isolation," Soil Dynamics and Earthquake Engineering, vol. 31, no. 3, pp. 535-545, 2011.

[21] H. Huang and G. C. Gaunaurd, "Acoustic scattering of a plane wave by two spherical elastic shells," The Journal of the Acoustical Society of America, vol. 98, no. 4, pp. 2149-2156, 1995.

[22] H. Huang and G. C. Gaunaurd, "Acoustic scattering of a plane wave by two spherical elastic shells above the coincidence frequency," The Journal of the Acoustical Society of America, vol. 101, no. 5, pp. 2659-2668, 1997.

[23] S. Robert, J.-M. Conoir, H. Franklin, and F. Luppé, "Resonant elastic scattering by a finite number of cylindrical cavities in an elastic matrix," Wave Motion, vol. 40, no. 3, pp. 225-239, 2004.

[24] P.-Y. le Bas, F. Luppé, J.-M. Conoir, and H. Franklin, "N-shell cluster in water: multiple scattering and splitting of resonances," The Journal of the Acoustical Society of America, vol. 115, no. 4, pp. 1460-1467, 2004.

[25] L. Rayleigh, "On the passage of waves through apertures in plane screens and allied problems," Philosophical Magazine, vol. 43, no. 263, pp. 259-272, 1897.

[26] A. C. Eringen and E. S. Suhubi, Elastodynamics, Academic Press, 1974.

[27] J. D. Achenbach, Wave Propagation in Elastic Solids, NorthHolland, Amsterdam, The Netherlands, 1973.

[28] Y. H. Pao and C. C. Mow, Diffraction of Elastic Waves and Dynamic Stress Concentrations, Crane and Russak, New York, NY, USA, 1973.

[29] M. M. Sun, Research on multiple scattering of elastic waves by rows of discontinuous barrier [Ph.D. thesis], Zhejiang University, 2012.

[30] G. N. Watson, A Treatise on the Theory of Bessel Functions, Cambridge University Press, Oxford, UK, 1966. 

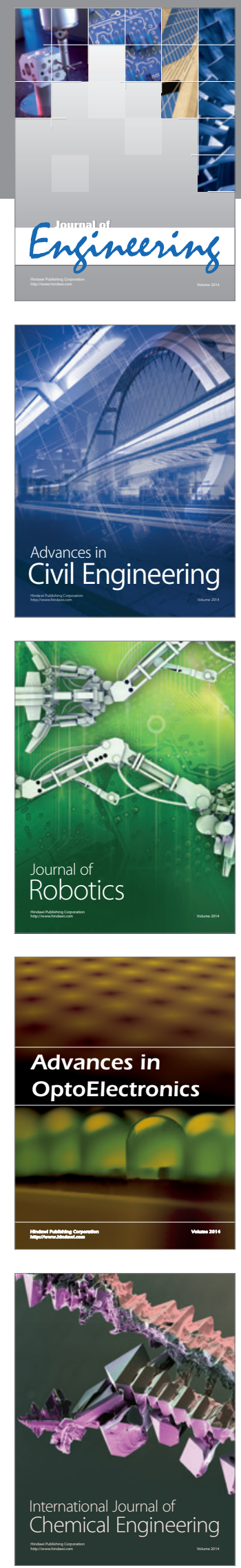

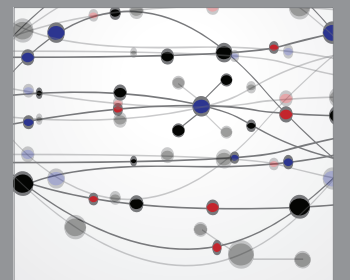

The Scientific World Journal
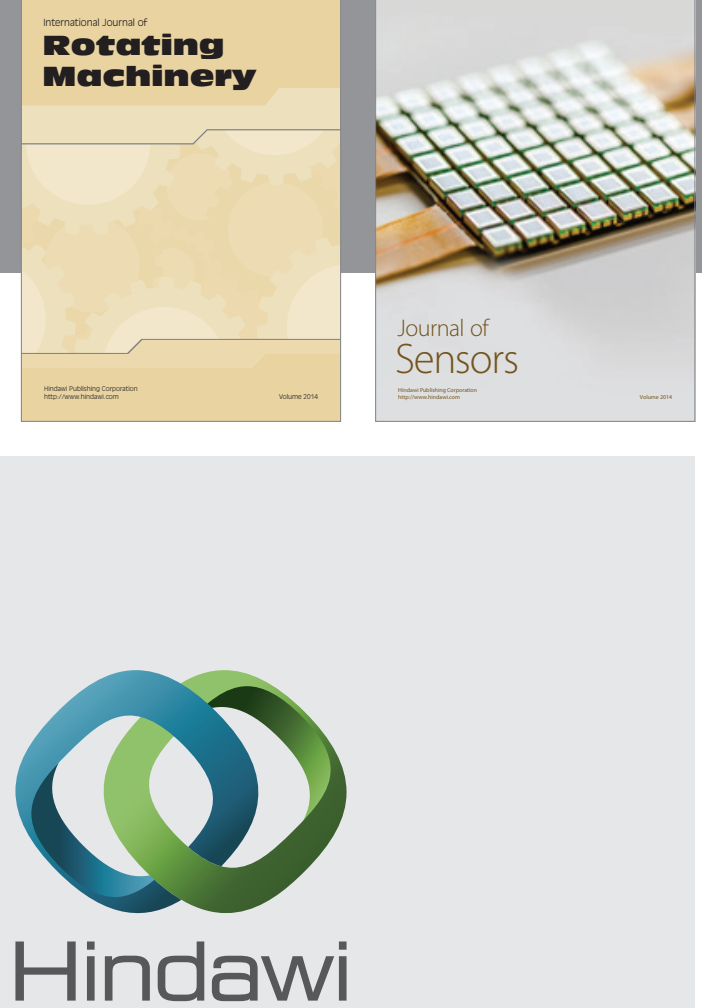

Submit your manuscripts at http://www.hindawi.com
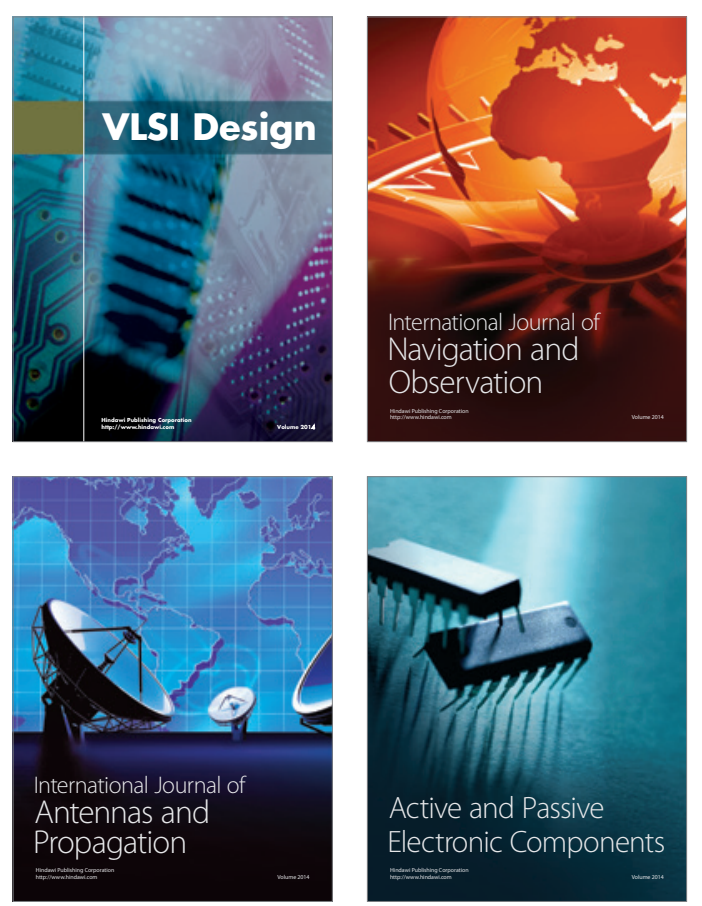
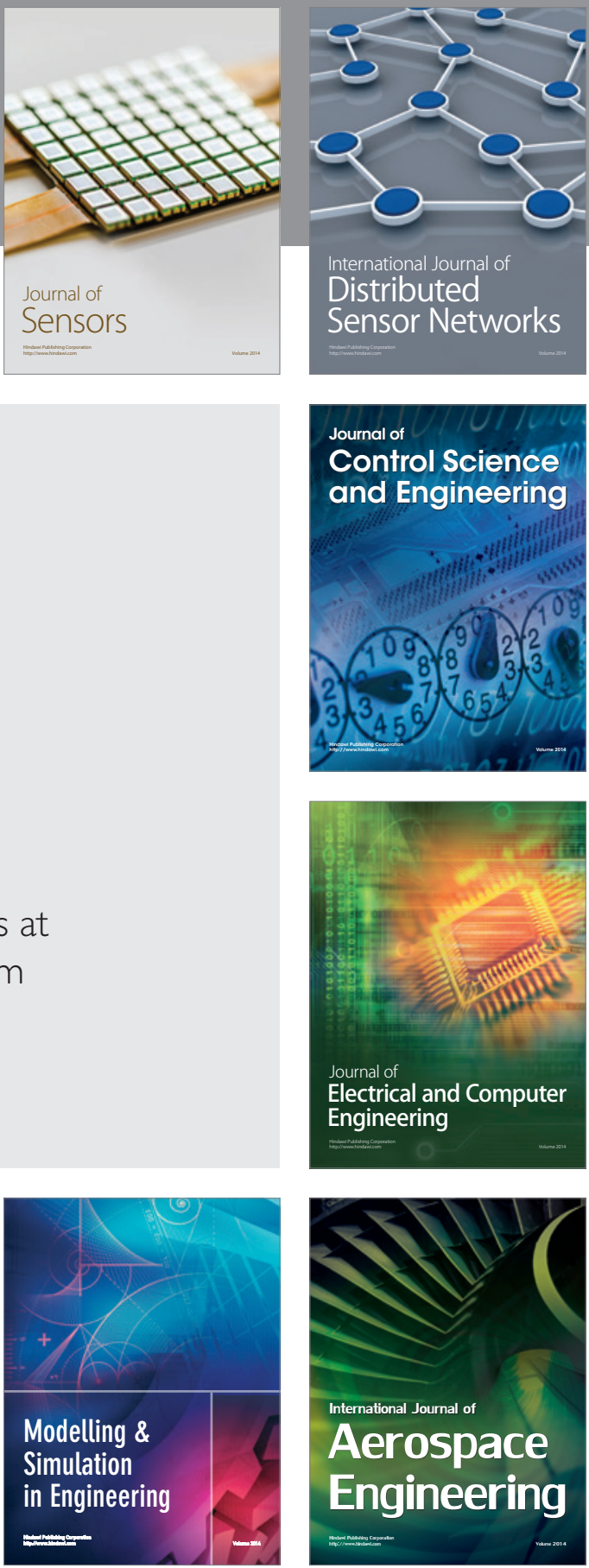

Journal of

Control Science

and Engineering
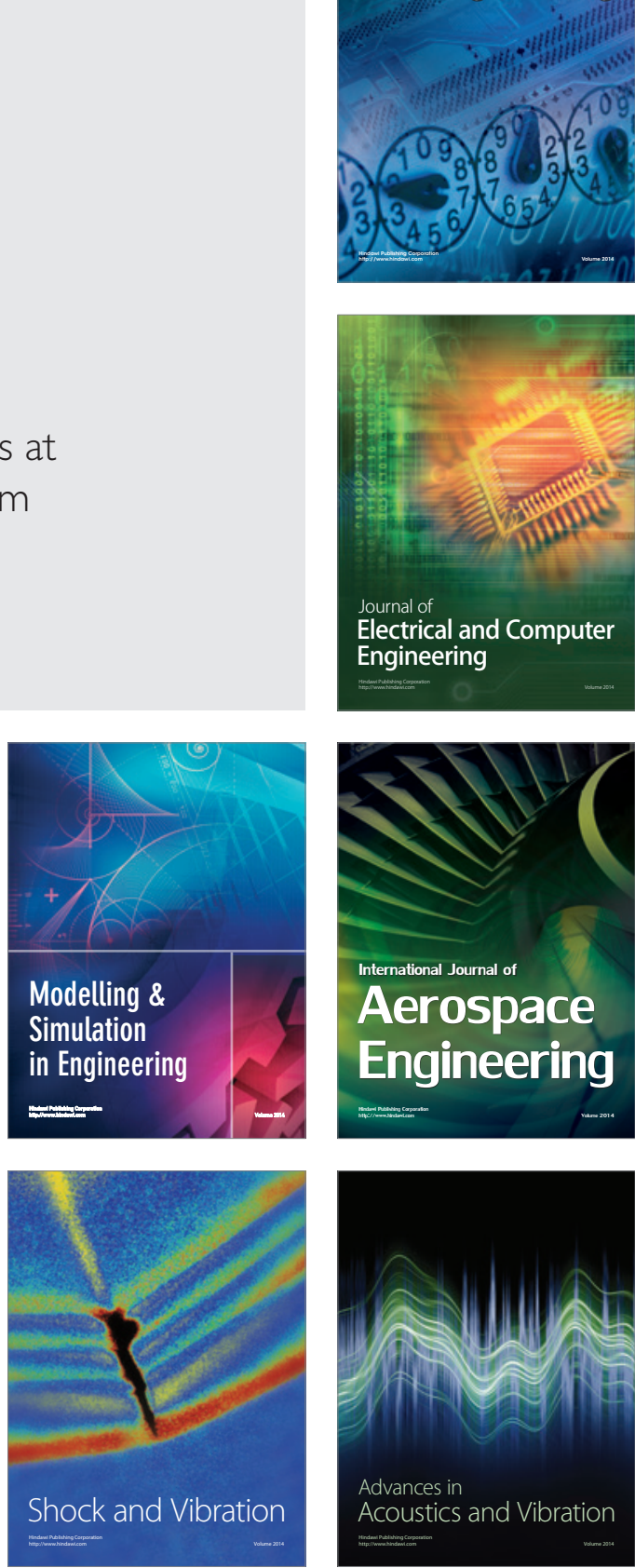